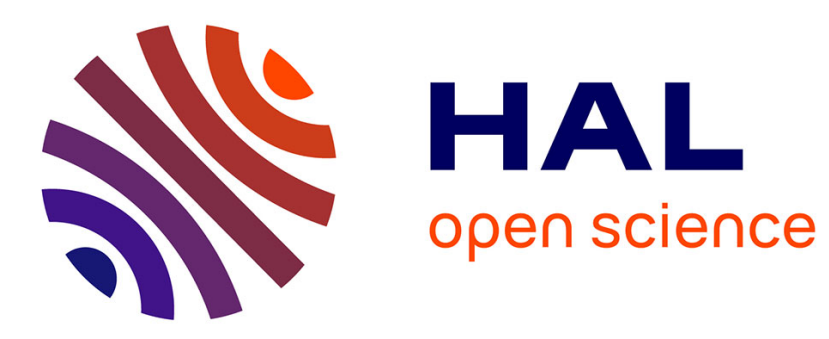

\title{
Speckle observations with PISCO in Merate. VI. Astrometric measurements of visual binaries in 2006
}

Marco Scardia, Jean-Louis Prieur, Luigi Pansecchi, Robert W. Argyle, Marco Sala

\section{- To cite this version:}

Marco Scardia, Jean-Louis Prieur, Luigi Pansecchi, Robert W. Argyle, Marco Sala. Speckle observations with PISCO in Merate. VI. Astrometric measurements of visual binaries in 2006. Astronomical Notes / Astronomische Nachrichten, 2009, 330, pp.55-67. 10.1002/asna.201913642 . hal-02460132

\section{HAL Id: hal-02460132 \\ https://hal.science/hal-02460132}

Submitted on 29 Jan 2020

HAL is a multi-disciplinary open access archive for the deposit and dissemination of scientific research documents, whether they are published or not. The documents may come from teaching and research institutions in France or abroad, or from public or private research centers.
L'archive ouverte pluridisciplinaire HAL, est destinée au dépôt et à la diffusion de documents scientifiques de niveau recherche, publiés ou non, émanant des établissements d'enseignement et de recherche français ou étrangers, des laboratoires publics ou privés. 


\title{
Speckle observations with PISCO in Merate. VI. Astrometric measurements of visual binaries in 2006
}

\author{
M. Scardia ${ }^{1}$, J.-L. Prieur ${ }^{2}$, L. Pansecchi ${ }^{1}$, R.W. Argyle ${ }^{3}$, and M. Sala ${ }^{1}$ \\ 1 INAF - Osservatorio Astronomico di Brera, Via E. Bianchi 46, 23807 Merate, Italia \\ 2 Laboratoire d'Astrophysique de Toulouse-Tarbes, Université de Toulouse, CNRS, 14 avenue E. Belin, 31400 \\ Toulouse, France \\ 3 Institute of Astronomy, Madingley Road, Cambridge, CB3 0HA, United Kingdom
}

Received August 14, 2008; accepted

Key words Stars: binaries: close - binaries: visual — astrometry — techniques: interferometric — individual (ADS 11479, ADS 11584, ADS 16538)

\begin{abstract}
We present relative astrometric measurements of visual binaries made during the second semester of 2006, with the speckle camera PISCO at the $102 \mathrm{~cm}$ Zeiss telescope of Brera Astronomical Observatory, in Merate. Our sample contains orbital couples as well as binaries whose motion is still uncertain. We obtained 175 new measurements of 169 objects, with angular separations in the range $0^{\prime \prime} .1-4^{\prime \prime} .2$, and an average accuracy of $0^{\prime \prime} .01$. The mean error on the position angles is $0^{\circ} .6$. Most of the position angles could be determined without the usual $180^{\circ}$ ambiguity with the application of triple-correlation techniques and/or by inspection of the long integration files.

We also present the new orbits we have computed for ADS 11479, 11584 and 16538, for which our measurements lead to large residuals and/or for which the revision was justified by the significant number of observations made since the last orbit computation.
\end{abstract}

(C) 0000 WILEY-VCH Verlag GmbH \& Co. KGaA, Weinheim

\section{Introduction}

This paper is the sixth of a series (Scardia et al. 2005, 2006, 2007, 2008a, Prieur et al. 2008, herein: Papers I to $\mathrm{V}$ ), whose purpose is to contribute to the determination of binary orbits, using speckle observations made in Merate (Italy) with the Pupil Interferometry Speckle camera and COronagraph (PISCO) on the $102 \mathrm{~cm}$ Zeiss telescope of INAF - Osservatorio Astronomico di Brera (OAB, Brera Astronomical Observatory). PISCO was developed at Observatoire Midi-Pyrénées and first used at Pic du Midi from 1993 to 1998. It was moved to Merate in 2004 and used there since. More information about the context and the purpose of this program can be found in Paper I.

This paper deals with the results of the observations performed during the second semester of 2006. In Sect. 2, we briefly describe our sample, the instrumental setup and the reduction procedure. The astrometric measurements are presented and discussed in Sect. 3. In Sect. 4 we propose new revised orbits for ADS 11479, 11584 and 16538 , partly derived from those observations and derive estimates of the component masses.

\section{Observations and data reduction}

The purpose of our long term program is to monitor the relative motion of all visual binaries accessible with
PISCO on the Zeiss telescope in Merate, for which new measurements are needed to improve their orbits. Our sample consists of visual binaries with the following characteristics, that are linked to instrumental or atmospheric limitations:

- declination north of $-5^{\circ}$,

- brighter than 10th magnitude in $V$,

- magnitude difference less than 4 ,

- angular separation smaller than $\approx 5^{\prime \prime}$.

The last limitation was chosen so that the binary systems fit inside the isoplanatic patch of the atmosphere, which is a theoretical necessary condition for speckle measurements. The distribution of the angular separations measured in this paper is displayed in Fig. 1 and shows a maximum for $\rho \approx 0^{\prime \prime} .7$. The closest separation that can be measured with PISCO in Merate is of the order of the diffraction limit, which is $\lambda / D \approx 0^{\prime \prime} .13$ with the $R$ filter (i.e. $\lambda=650 \mathrm{~nm}$ ) and the Zeiss telescope whose diameter is $D=1.02 \mathrm{~m}$.

The observations were carried out with the PISCO speckle camera with the ICCD detector (CCD intensified with a micro-channel plate) belonging to Nice University (France). Details about the telescope and the instrumentation can be found in Paper I and in Prieur et al. (1998). For each observation, a series of about 10000 short-exposure frames were digitized and processed in real-time with a Pentium III PC, to compute the mean auto-correlation with Worden's (1977) 


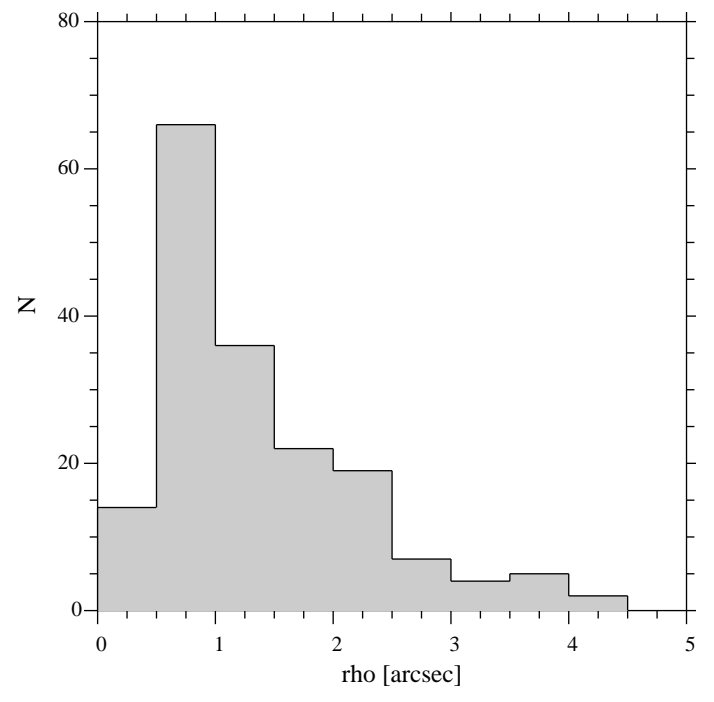

Fig. 1 Histogram of the angular separations of the 175 measurements reported in this paper.

method (which subtracts most of the continuum), the mean power spectrum and the integration of the individual frames. Those frames were also recorded on a SVHS video tape for archiving and further processing such as the quadrant determination.

As the auto-correlation function is symmetric relative to the origin, it does not contain information about the location of the faintest companion. This is the origin of the well-known $180^{\circ}$ ambiguity of binary speckle measurements. This ambiguity can be resolved by using the mean triple-correlation function of the elementary frames (Weigelt, 1977) or a restricted version of this function as proposed by Aristidi et al. (1997), which is the method we use (see Paper III).

The positions of the secondary peaks of the mean auto-correlations were carefully measured with an interactive program that fitted and subtracted the residual background. The details of this procedure with an evaluation of the reliability of the determination of the errors can be found in Paper III. For the binaries with the smallest separations (i.e. $\rho \lesssim 0^{\prime \prime} .3$ ) we also subtracted a model of the central background pattern to the auto-correlation images, as described in Paper IV.

\section{Astrometric measurements}

The astrometric measurements of the observations made during the second semester of 2006 are displayed in Table 1. The designation of the binary is reported in the first three columns: the WDS name (Washington Double Star Catalogue, Mason et al. 2007) in Col. 1, the official double star designation in Col. 2 (sequence is "discoverer-number"), and the ADS number in Col. 3 (Aitken, 1932). For each observation, we then give the epoch in Besselian years (Col. 4), the filter (Col. 5), the focal length of the eyepiece used for magnifying the image (Col. 6), the angular separation $\rho$ (Col. 7) with its error (Col. 8) in arcseconds, and the position angle $\theta$ (Col. 9) with its error (Col. 10) in degrees.

The characteristics of the PISCO $R$ and $V$ filters were given in Table 1 of Paper III. Some objects, like ADS 9426 and 10949, were observed without any filter because they were too faint. This is indicated with $W$ (for "white" light) in the filter column (Col. 5). The corresponding bandpass is that of the ICCD detector, with a central wavelength of about $650 \mathrm{~nm}$, close to that of the $R$ filter.

The errors reported in Cols. 8 and 10 were computed by adding quadratically the calibration errors to the standard deviations of series of measurements obtained with the same data sets (see Paper III). As for the previous papers of this series, the minimum (onesigma) errors for the angular separation (Col. 8) were set to $0^{\prime \prime} .003$ for close pairs and to $0.05 \%$ of $\rho$ for wide pairs, on the basis of the uncertainties coming from the determination of the centres of the auto-correlation peaks (estimated at 0.1 pixel in the elementary frames) and those affecting the scale calibration (i.e., 0.05\%), respectively. Similarly, the minimum (one-sigma) errors for the position angle (Col. 10) were estimated at $0^{\circ} .3$. The average values of the errors of the 175 measurements reported in this table are $0^{\prime \prime} .012 \pm 0^{\prime \prime} .005$ and $0^{\circ} .56 \pm 0^{\circ} .34$ for $\rho$ and $\theta$, respectively. The validity of our error determination was studied in detail in $\mathrm{Pa}-$ per III.

The position angles presented in Col. 9 follow the standard convention with the North corresponding to $\theta=0^{\circ}$ and the East to $\theta=90^{\circ}$. When the triple correlation files allowed us to resolve the $180^{\circ}$ ambiguity (see Sect. 2 and 3.1), an asterisk was added in Col. 9 to indicate that our determination is absolute. Otherwise, our angular measurements were reduced to the quadrant reported in the "Fourth Catalogue of Interferometric Measurements of Binary Stars" (Hartkopf et al. 2008, hereafter IC4).

In Col. 11, a flag is set to one for all the systems for which an orbit was found in the literature, e.g., mainly from the "Sixth Catalogue of Orbits of Visual Binary Stars" (Hartkopf \& Mason, 2008), hereafter OC6. The residuals derived from the corresponding ephemerides will be discussed in Sect. 3.2.

In the note column (Col. 12), we give some comments about the secondary peaks of the autocorrelation files that have been measured (e.g., elongated, diffuse, faint, etc). An elongation of those peaks is generally a sign of an insufficient correction of the atmospheric dispersion by the Risley prisms (see Prieur et al., 1998). The auto-correlation files of ADS 13447 exhibited some spurious multiple peaks whose origin is unknown. 
Table 1 Measurements of visual binaries between July and December 2006 (begin.).

\begin{tabular}{|c|c|c|c|c|c|c|c|c|c|c|c|}
\hline WDS & Name & $\mathrm{ADS}$ & Epoch & Fil. & $\begin{array}{l}\text { Eyep. } \\
(\mathrm{mm})\end{array}$ & $\begin{array}{c}\rho \\
(\operatorname{arcsec})\end{array}$ & $\begin{array}{c}\sigma_{\rho} \\
(\operatorname{arcsec})\end{array}$ & $\begin{array}{c}\theta \\
\text { (deg.) }\end{array}$ & $\begin{array}{c}\sigma_{\theta} \\
\text { (deg.) }\end{array}$ & Orb. & Notes \\
\hline $01283+4247$ & AC 14 & 1161 & 2006.954 & $\mathrm{R}$ & 20 & 0.768 & 0.019 & $92.1^{*}$ & 0.5 & 0 & \\
\hline $14536-0028$ & BU 942 & 9426 & 2006.499 & W & 20 & 1.388 & 0.028 & 185.7 & 1.3 & 0 & \\
\hline $15361+5531$ & A 1124 & 9720 & 2006.499 & $\mathrm{R}$ & 20 & 1.399 & 0.027 & $141.0^{*}$ & 1.1 & 0 & \\
\hline $16413+3136$ & STF 2084 & 10157 & 2006.642 & $\mathrm{R}$ & 10 & 1.019 & 0.005 & $208.7^{*}$ & 0.5 & 1 & \\
\hline $16442+2331$ & STF 2094AB & 10184 & 2006.655 & $\mathrm{R}$ & 20 & 1.185 & 0.008 & $72.7^{*}$ & 0.6 & 0 & \\
\hline $16518+2840$ & STF 2107AB & 10235 & 2006.680 & $\mathrm{R}$ & 20 & 1.452 & 0.008 & $99.6^{*}$ & 0.3 & 1 & \\
\hline $16541+0826$ & HEI 857 & - & 2006.680 & $\mathrm{R}$ & 20 & 0.563 & 0.008 & $142.2^{*}$ & 0.5 & 0 & \\
\hline $16564+6502$ & STF 2118AB & 10279 & 2006.639 & $\mathrm{R}$ & 20 & 1.067 & 0.008 & $66.2^{*}$ & 0.3 & 1 & \\
\hline $17020+0827$ & STF 2114 & 10312 & 2006.674 & $\mathrm{R}$ & 20 & 1.343 & 0.008 & $193.8^{*}$ & 0.3 & 0 & \\
\hline $17053+5428$ & STF 2130AB & 10345 & 2006.639 & $\mathrm{R}$ & 20 & 2.360 & 0.012 & $11.8^{*}$ & 0.3 & 1 & \\
\hline $17096+0356$ & HEI 894 & - & 2006.696 & $\mathrm{R}$ & 20 & 0.553 & 0.009 & $21.8^{*}$ & 0.8 & 0 & \\
\hline $17195+5832$ & KR 46 & 10487 & 2006.642 & $\mathrm{R}$ & 20 & 1.695 & 0.010 & $63.3^{*}$ & 0.6 & 0 & \\
\hline $17239+3627$ & STF 2162 & 10527 & 2006.674 & $\mathrm{R}$ & 20 & 1.355 & 0.010 & 282.2 & 0.7 & 0 & \\
\hline $17240+3835$ & HU 1179 & 10531 & 2006.675 & $\mathrm{R}$ & 10 & 0.247 & 0.006 & 274.4 & 0.3 & 1 & \\
\hline $17304-0104$ & STF 2173 & 10598 & 2006.680 & $\mathrm{R}$ & 10 & 0.451 & 0.003 & $166.2^{*}$ & 0.3 & 1 & Elongated \\
\hline $17358+0100$ & STF 2186 & 10650 & 2006.639 & $\mathrm{R}$ & 20 & 3.022 & 0.015 & $78.2^{*}$ & 0.3 & 0 & \\
\hline $17386+5546$ & STF 2199 & 10699 & 2006.694 & $\mathrm{R}$ & 20 & 2.007 & 0.010 & $56.5^{*}$ & 0.3 & 1 & \\
\hline $17397+7256$ & H 141 & 10734 & 2006.639 & $\mathrm{R}$ & 20 & 1.036 & 0.008 & $336.0^{*}$ & 0.4 & 0 & \\
\hline $17399+0748$ & HDS2499 & - & 2006.639 & $\mathrm{R}$ & 20 & 0.680 & 0.008 & $239.3^{*}$ & 0.9 & 0 & \\
\hline $17436+2237$ & HU 1285 & 10743 & 2006.677 & $\mathrm{R}$ & 20 & 0.546 & 0.008 & $215.0^{*}$ & 0.4 & 1 & \\
\hline $17439+0551$ & STF 2200AB & 10741 & 2006.677 & $\mathrm{R}$ & 20 & 1.594 & 0.008 & $162.8^{*}$ & 0.3 & 0 & \\
\hline $17457+1743$ & STF 2205 & 10769 & 2006.696 & $\mathrm{R}$ & 20 & 1.076 & 0.008 & $358.3^{*}$ & 0.3 & 1 & \\
\hline $17471+1742$ & STF 2215 & 10795 & 2006.677 & $\mathrm{R}$ & 10 & 0.505 & 0.003 & $254.8^{*}$ & 0.3 & 1 & \\
\hline $17533+3605$ & STF 2243 & 10874 & 2006.642 & $\mathrm{R}$ & 20 & 1.141 & 0.014 & 39.8 & 0.6 & 0 & \\
\hline $17571+0004$ & STF 2244 & 10912 & 2006.680 & $\mathrm{R}$ & 10 & 0.611 & 0.003 & $97.8^{*}$ & 0.4 & 1 & \\
\hline $17571+4551$ & HU 235 & 10934 & 2006.680 & $\mathrm{R}$ & 20 & 1.608 & 0.008 & $282.9^{*}$ & 0.3 & 0 & \\
\hline $17590+0202$ & STF $2252 \mathrm{AB}$ & 10945 & 2006.696 & $\mathrm{R}$ & 20 & 3.958 & 0.020 & 23.5 & 0.3 & 0 & \\
\hline $17590+1226$ & STF $2254 \mathrm{AB}$ & 10949 & 2006.675 & $\mathrm{~W}$ & 20 & 3.502 & 0.018 & $265.6^{*}$ & 0.3 & 0 & Diffuse \\
\hline $18003+5251$ & STF $2271 \mathrm{AB}$ & 10988 & 2006.677 & $\mathrm{R}$ & 20 & 3.395 & 0.017 & $267.1^{*}$ & 0.3 & 0 & \\
\hline $18017+4011$ & STF 2267 & 11001 & 2006.642 & $\mathrm{R}$ & 20 & 0.567 & 0.008 & 269.9 & 0.5 & 0 & \\
\hline $18025+4414$ & BU $1127 \mathrm{Aa}-\mathrm{B}$ & 11010 & 2006.639 & $\mathrm{R}$ & 20 & 0.775 & 0.008 & $55.2^{*}$ & 0.7 & 1 & \\
\hline $18065+4022$ & STF 2282 & 11074 & 2006.639 & $\mathrm{R}$ & 20 & 2.657 & 0.013 & $81.8^{*}$ & 0.4 & 0 & \\
\hline $18096+0400$ & STF $2281 \mathrm{AB}$ & 11111 & 2006.718 & $\mathrm{~W}$ & 10 & 0.602 & 0.010 & $291.0^{*}$ & 0.7 & 1 & \\
\hline$"$ & $"$ & $"$ & 2006.721 & $\mathrm{R}$ & 20 & 0.588 & 0.008 & $290.8^{*}$ & 1.0 & 1 & \\
\hline $18097+5024$ & HU 674 & 11128 & 2006.677 & $\mathrm{R}$ & 20 & 0.753 & 0.008 & $216.1^{*}$ & 0.7 & 1 & \\
\hline $18101+1629$ & STF 2289 & 11123 & 2006.694 & $\mathrm{R}$ & 20 & 1.231 & 0.008 & $219.2^{*}$ & 0.3 & 1 & \\
\hline $18146+0011$ & STF 2294 & 11186 & 2006.696 & $\mathrm{R}$ & 20 & 1.295 & 0.014 & 92.6 & 0.6 & 1 & \\
\hline $18208+7120$ & STT 353AB & 11311 & 2006.699 & $\mathrm{R}$ & 10 & 0.480 & 0.004 & $267.6^{*}$ & 0.3 & 1 & \\
\hline $18238+5139$ & ES 187AB & 11328 & 2006.642 & $\mathrm{R}$ & 20 & 2.561 & 0.014 & 206.0 & 0.3 & 0 & \\
\hline $18250-0135$ & $\mathrm{AC} 11$ & 11324 & 2006.696 & $\mathrm{R}$ & 20 & 0.873 & 0.008 & $354.7^{*}$ & 0.3 & 1 & \\
\hline $18250+2724$ & STF 2315AB & 11334 & 2006.696 & $\mathrm{R}$ & 10 & 0.642 & 0.003 & 121.2 & 0.3 & 1 & \\
\hline $18261+0047$ & BU 1203 & 11339 & 2006.726 & $\mathrm{R}$ & 20 & 0.459 & 0.009 & $153.9^{*}$ & 0.5 & 1 & \\
\hline $18272+0012$ & STF 2316Aa-B & 11353 & 2006.726 & $\mathrm{R}$ & 20 & 3.681 & 0.018 & $319.8^{*}$ & 0.3 & 0 & \\
\hline $18320+0647$ & STT 354 & 11432 & 2006.699 & $\mathrm{R}$ & 20 & 0.610 & 0.008 & 210.8 & 1.3 & 0 & \\
\hline $18338+1744$ & STF 2339AB-D & 11454 & 2006.699 & $\mathrm{R}$ & 20 & 2.081 & 0.010 & $273.5^{*}$ & 0.4 & 0 & (outer autoc. peaks) \\
\hline$"$ & $"$ & $"$ & 2006.716 & $\mathrm{R}$ & 20 & 2.058 & 0.013 & $273.7^{*}$ & 0.4 & 0 & \\
\hline $18338+1744$ & STF 2339AB-C & 11454 & 2006.699 & $\mathrm{R}$ & 20 & 1.632 & 0.014 & $276.4^{*}$ & 0.4 & 0 & (inner autoc. peaks) \\
\hline$"$ & $"$ & $"$ & 2006.715 & $\mathrm{~W}$ & 20 & 1.640 & 0.008 & $275.3^{*}$ & 0.5 & 0 & \\
\hline$"$ & $"$ & $"$ & 2006.716 & $\mathrm{R}$ & 20 & 1.627 & 0.008 & $275.8^{*}$ & 0.4 & 0 & \\
\hline$"$ & $"$ & $"$ & 2006.715 & $\mathrm{~W}$ & 10 & - & - & - & - & 0 & Too diffuse \\
\hline $18338+1744$ & WAK 21CD & 11454 & 2006.699 & $\mathrm{R}$ & 20 & 0.441 & 0.018 & $265.5^{*}$ & 0.5 & 0 & \\
\hline $18339+5221$ & A $1377 \mathrm{AB}$ & 11468 & 2006.677 & $\mathrm{R}$ & 10 & 0.251 & 0.005 & $120.7^{*}$ & 0.6 & 1 & Elongated \\
\hline $18355+2336$ & STT 359 & 11479 & 2006.639 & $\mathrm{R}$ & 10 & 0.729 & 0.004 & 5.6 & 0.3 & 1 & \\
\hline $18359+1659$ & STT 358AB & 11483 & 2006.675 & $\mathrm{R}$ & 20 & 1.655 & 0.008 & $151.8^{*}$ & 0.3 & 1 & \\
\hline $18374+7741$ & STT 363 & 11584 & 2006.727 & $\mathrm{R}$ & 20 & 0.431 & 0.013 & 336.9 & 0.7 & 1 & \\
\hline $18384+2842$ & STF 2356 & 11529 & 2006.642 & $\mathrm{R}$ & 20 & 1.125 & 0.010 & 62.8 & 0.5 & 0 & \\
\hline
\end{tabular}


Table 1 Measurements of visual binaries between July and December 2006 (cont.).

\begin{tabular}{|c|c|c|c|c|c|c|c|c|c|c|c|}
\hline WDS & Name & ADS & Epoch & Fil. & $\begin{array}{l}\text { Eyep. } \\
(\mathrm{mm})\end{array}$ & $\begin{array}{c}\rho \\
(\operatorname{arcsec})\end{array}$ & $\begin{array}{c}\sigma_{\rho} \\
(\operatorname{arcsec})\end{array}$ & $\begin{array}{c}\theta \\
\text { (deg.) }\end{array}$ & $\begin{array}{c}\sigma_{\theta} \\
\text { (deg.) }\end{array}$ & Orb. & Notes \\
\hline $18387+0451$ & STT 360 & 11526 & 2006.743 & $\mathrm{R}$ & 20 & 1.672 & 0.008 & $281.4^{*}$ & 0.3 & 0 & \\
\hline $18393+2056$ & STF 2360 & 11546 & 2006.677 & $\mathrm{R}$ & 20 & 2.432 & 0.012 & $358.3^{*}$ & 0.3 & 0 & \\
\hline $18413+3018$ & STF 2367AB & 11579 & 2006.743 & $\mathrm{R}$ & 10 & 0.378 & 0.003 & 75.4 & 0.5 & 1 & \\
\hline $18443+3940$ & STF $2382 \mathrm{AB}$ & 11635 & 2006.675 & $\mathrm{R}$ & 20 & 2.380 & 0.012 & $348.8^{*}$ & 0.3 & 1 & \\
\hline $18443+3940$ & STF 2383 Cc-D & 11635 & 2006.675 & $\mathrm{R}$ & 20 & 2.381 & 0.012 & $79.5^{*}$ & 0.3 & 1 & \\
\hline $18443+6103$ & STF 2403 & 11661 & 2006.680 & $\mathrm{R}$ & 20 & 1.091 & 0.008 & $277.2^{*}$ & 0.3 & 0 & \\
\hline $18477+4904$ & HEI 72 & - & 2006.724 & $\mathrm{R}$ & 20 & 0.618 & 0.008 & $231.4^{*}$ & 0.6 & 0 & \\
\hline $18490+2110$ & STF $2401 \mathrm{AB}$ & 11715 & 2006.724 & W & 20 & 4.248 & 0.021 & $37.7^{*}$ & 0.3 & 0 & \\
\hline $18497+1041$ & STF 2402 & 11722 & 2006.784 & $\mathrm{R}$ & 20 & 1.455 & 0.008 & $208.8^{*}$ & 0.6 & 0 & \\
\hline $18502+1131$ & BU 265 & 11735 & 2006.784 & $\mathrm{R}$ & 20 & 1.390 & 0.013 & $228.4^{*}$ & 0.3 & 0 & \\
\hline $18508+1059$ & STF 2404 & 11750 & 2006.694 & $\mathrm{R}$ & 20 & 3.534 & 0.018 & $181.2^{*}$ & 0.3 & 0 & \\
\hline $18520+1047$ & STF 2408 & 11766 & 2006.784 & $\mathrm{~W}$ & 20 & 2.286 & 0.011 & $90.7^{*}$ & 0.3 & 0 & Elongated \\
\hline $18521+1148$ & HU 199 & 11769 & 2006.789 & $\mathrm{R}$ & 20 & 0.884 & 0.011 & $345.6^{*}$ & 1.0 & 0 & \\
\hline $18526+1400$ & STF 2412 & 11778 & 2006.694 & $\mathrm{R}$ & 20 & 1.449 & 0.008 & 55.9 & 0.3 & 0 & \\
\hline $18555+2914$ & STF 2419 & 11847 & 2006.789 & $\mathrm{R}$ & 20 & 3.363 & 0.017 & $176.9^{*}$ & 0.3 & 0 & \\
\hline $18570+3254$ & $\mathrm{BU} 648 \mathrm{AB}$ & 11871 & 2006.721 & $\mathrm{R}$ & 20 & 0.898 & 0.008 & $268.8^{*}$ & 0.3 & 1 & \\
\hline $18575+5814$ & STF 2438 & 11897 & 2006.716 & $\mathrm{R}$ & 10 & 0.867 & 0.008 & $359.6^{*}$ & 0.3 & 1 & \\
\hline $19019+1910$ & STF 2437 & 11956 & 2006.743 & $\mathrm{R}$ & 20 & 0.580 & 0.016 & 11.1 & 0.3 & 1 & \\
\hline $19024+6927$ & STF 2478 & 12015 & 2006.680 & $\mathrm{~W}$ & 20 & 0.953 & 0.008 & $313.6^{*}$ & 0.5 & 0 & \\
\hline $19030+5135$ & STF 2451 & 11997 & 2006.677 & $\mathrm{~W}$ & 20 & 1.984 & 0.010 & $81.9^{*}$ & 0.4 & 0 & \\
\hline $19052+1050$ & BU 466 & 12021 & 2006.669 & $\mathrm{~W}$ & 20 & 1.916 & 0.010 & $163.8^{*}$ & 0.3 & 0 & \\
\hline $19062+3026$ & STF $2454 \mathrm{AB}$ & 12040 & 2006.822 & $\mathrm{~W}$ & 20 & 1.326 & 0.019 & $288.6^{*}$ & 0.5 & 1 & \\
\hline $19070+1104$ & HEI 568 & - & 2006.724 & $\mathrm{R}$ & 10 & 0.304 & 0.004 & $274.9^{*}$ & 0.4 & 0 & \\
\hline $19071+7204$ & STT 369 & 12113 & 2006.721 & $\mathrm{R}$ & 20 & 0.695 & 0.011 & $11.3^{*}$ & 0.5 & 0 & \\
\hline $19083+5520$ & D $19 A B$ & 12104 & 2006.727 & $\mathrm{R}$ & 20 & 0.511 & 0.016 & $348.6^{*}$ & 1.1 & 0 & \\
\hline $19114+2116$ & A 151 & 12140 & 2006.819 & $\mathrm{R}$ & 20 & 0.574 & 0.011 & 156.2 & 0.7 & 0 & \\
\hline $19143+1904$ & STF 2484 & 12201 & 2006.694 & $\mathrm{R}$ & 20 & 2.163 & 0.011 & $238.9^{*}$ & 0.3 & 1 & \\
\hline $19148+4756$ & A 706 & 12229 & 2006.789 & $\mathrm{R}$ & 20 & 1.600 & 0.014 & 73.4 & 0.5 & 0 & \\
\hline $19220+2230$ & $\mathrm{BU} 141 \mathrm{AB}$ & 12355 & 2006.694 & $\mathrm{R}$ & 20 & 0.895 & 0.008 & 82.1 & 0.9 & 0 & \\
\hline $19251+1839$ & HU 339 & 12416 & 2006.792 & $\mathrm{~W}$ & 20 & 0.834 & 0.008 & 243.5 & 0.7 & 0 & Diffuse \& elongated \\
\hline $19261+3849$ & $\mathrm{HO} 450 \mathrm{AB}$ & 12446 & 2006.697 & $\mathrm{R}$ & 20 & 1.000 & 0.013 & 264.1 & 0.8 & 0 & \\
\hline $19266+2719$ & STF 2525 & 12447 & 2006.677 & $\mathrm{R}$ & 20 & 2.130 & 0.016 & $289.7^{*}$ & 0.3 & 1 & \\
\hline $19270+7322$ & STF $2550 \mathrm{AB}$ & 12524 & 2006.680 & $\mathrm{R}$ & 20 & 1.942 & 0.010 & 250.8 & 0.3 & 0 & \\
\hline $19299+4931$ & BU 143 & 12535 & 2006.697 & $\mathrm{~W}$ & 20 & 2.188 & 0.017 & $192.3^{*}$ & 0.3 & 0 & \\
\hline $19307+2758$ & MCA 55Aac & 12540 & 2006.721 & $\mathrm{R}$ & 10 & 0.359 & 0.012 & $105.5^{*}$ & 1.3 & 1 & \\
\hline $19311+0824$ & A 1184 & 12537 & 2006.743 & $\mathrm{~W}$ & 20 & 0.901 & 0.024 & $108.4^{*}$ & 0.4 & 0 & Elongated \\
\hline $19346+1808$ & STT 375 & 12623 & 2006.721 & $\mathrm{R}$ & 20 & 0.601 & 0.015 & $182.5^{*}$ & 1.2 & 0 & \\
\hline $19350+2947$ & A 368 & 12633 & 2006.699 & $\mathrm{R}$ & 20 & 0.507 & 0.011 & $153.2^{*}$ & 0.8 & 0 & \\
\hline $19357+7308$ & A 864 & 12729 & 2006.727 & $\mathrm{~W}$ & 20 & 0.742 & 0.008 & $14.5^{*}$ & 1.3 & 0 & \\
\hline $19363+3540$ & STT $377 \mathrm{AB}$ & 12667 & 2006.819 & $\mathrm{R}$ & 20 & 0.985 & 0.035 & 36.1 & 1.7 & 0 & \\
\hline $19365+4101$ & STT $378 \mathrm{AB}$ & 12687 & 2006.699 & $\mathrm{R}$ & 20 & 1.406 & 0.008 & $285.5^{*}$ & 0.3 & 0 & \\
\hline $19384+0021$ & BU $249 \mathrm{AB}$ & 12708 & 2006.852 & $\mathrm{R}$ & 20 & 0.831 & 0.017 & $109.9^{*}$ & 1.0 & 0 & \\
\hline $19402+2331$ & A 166 & 12770 & 2006.830 & $\mathrm{R}$ & 20 & 0.821 & 0.015 & 241.2 & 1.2 & 0 & \\
\hline$"$ & $"$ & & 2006.830 & $\mathrm{~W}$ & 20 & 0.805 & 0.017 & $62.4^{*}$ & 0.7 & 0 & \\
\hline $19413+3043$ & $\mathrm{BU} 145 \mathrm{AB}$ & 12786 & 2006.852 & $\mathrm{R}$ & 20 & 0.825 & 0.011 & $269.5^{*}$ & 0.8 & 0 & \\
\hline $19448+1649$ & STF 2569 & 12861 & 2006.830 & $\mathrm{R}$ & 20 & 2.119 & 0.015 & $357.0^{*}$ & 0.3 & 0 & \\
\hline $19450+4508$ & STF 2579AB & 12880 & 2006.721 & $\mathrm{R}$ & 20 & 2.666 & 0.013 & $220.4^{*}$ & 0.3 & 1 & \\
\hline$"$ & $"$ & $"$ & 2006.762 & $\mathrm{R}$ & 20 & 2.647 & 0.013 & $220.5^{*}$ & 0.3 & 1 & \\
\hline $19453+3048$ & AG 237 & 12881 & 2006.762 & $\mathrm{~W}$ & 20 & 2.375 & 0.018 & $140.2^{*}$ & 0.5 & 0 & \\
\hline $19456+3337$ & STF $2576 \mathrm{AB}$ & 12889 & 2006.699 & $\mathrm{R}$ & 20 & 2.832 & 0.014 & 160.4 & 0.3 & 1 & \\
\hline $19483+3710$ & STT 386 & 12965 & 2006.669 & $\mathrm{R}$ & 20 & 0.933 & 0.011 & 69.8 & 0.9 & 0 & \\
\hline $19487+3519$ & STT 387 & 12972 & 2006.724 & $\mathrm{R}$ & 20 & 0.571 & 0.018 & $129.6^{*}$ & 0.8 & 1 & \\
\hline $19575+2018$ & $\mathrm{BU} 425 \mathrm{AB}$ & 13165 & 2006.852 & $\mathrm{~W}$ & 20 & 1.342 & 0.026 & 240.0 & 0.6 & 0 & Elongated \\
\hline $20011+4816$ & STF $2619 \mathrm{AB}$ & 13269 & 2006.784 & $\mathrm{R}$ & 20 & 4.166 & 0.021 & $239.3^{*}$ & 0.3 & 0 & \\
\hline $20014+1045$ & STF 2613 & 13256 & 2006.727 & $\mathrm{R}$ & 20 & 3.637 & 0.018 & $354.2^{*}$ & 0.3 & 1 & \\
\hline
\end{tabular}


Table 1 Measurements of visual binaries between July and December 2006 (cont.).

\begin{tabular}{|c|c|c|c|c|c|c|c|c|c|c|c|}
\hline WDS & Name & ADS & Epoch & Fil. & $\begin{array}{l}\text { Eyep. } \\
(\mathrm{mm})\end{array}$ & $\begin{array}{c}\rho \\
(\operatorname{arcsec})\end{array}$ & $\begin{array}{c}\sigma_{\rho} \\
(\operatorname{arcsec})\end{array}$ & $\begin{array}{c}\theta \\
\text { (deg.) }\end{array}$ & $\begin{array}{c}\sigma_{\theta} \\
\text { (deg.) }\end{array}$ & Orb. & Notes \\
\hline $20042+1148$ & STF 2620AB & 13320 & 2006.830 & $\mathrm{R}$ & 20 & 1.849 & 0.009 & $286.1^{*}$ & 0.5 & 0 & \\
\hline $20067+1256$ & BU 428 & 13384 & 2006.727 & $\mathrm{R}$ & 20 & 0.788 & 0.008 & 353.6 & 0.7 & 0 & \\
\hline $20095+5140$ & STF 2645 & 13447 & 2006.789 & $\mathrm{R}$ & 20 & 1.594 & 0.010 & 138.5 & 0.8 & 0 & Multiple peaks \\
\hline $20102+4357$ & STT 400 & 13461 & 2006.699 & $\mathrm{R}$ & 20 & 0.593 & 0.008 & 337.4 & 0.7 & 1 & \\
\hline $20187+3315$ & $\mathrm{STT} 405 \mathrm{AB}$ & 13682 & 2006.819 & $\mathrm{R}$ & 20 & 0.799 & 0.017 & 150.6 & 0.7 & 0 & \\
\hline $20200+3616$ & BU 431 & 13719 & 2006.820 & $\mathrm{R}$ & 20 & 0.530 & 0.008 & $29.8^{*}$ & 1.8 & 0 & \\
\hline $20229+4259$ & HO $128 \mathrm{AB}$ & 13786 & 2006.820 & $\mathrm{R}$ & 20 & 1.361 & 0.021 & $358.8^{*}$ & 0.6 & 0 & \\
\hline $20244+2923$ & $\mathrm{HO} 457 \mathrm{AB}$ & 13818 & 2006.830 & $\mathrm{~W}$ & 20 & 2.021 & 0.010 & $60.4^{*}$ & 0.3 & 0 & Elongated \\
\hline $20248+3545$ & BU 432 & 13830 & 2006.831 & $\mathrm{R}$ & 20 & 1.433 & 0.019 & $196.5^{*}$ & 0.5 & 0 & \\
\hline $20337+3835$ & A 1431 & 14007 & 2006.789 & $\mathrm{R}$ & 20 & 0.867 & 0.011 & 30.6 & 0.6 & 0 & \\
\hline $20370+1203$ & STF 2701 & 14063 & 2006.697 & $\mathrm{R}$ & 20 & 2.057 & 0.020 & $221.1^{*}$ & 0.5 & 0 & \\
\hline $20375+1436$ & $\mathrm{BU} 151 \mathrm{AB}$ & 14073 & 2006.790 & $\mathrm{R}$ & 10 & 0.532 & 0.003 & $5.5^{*}$ & 0.3 & 1 & \\
\hline $20445+2356$ & STF 2724 & 14227 & 2006.784 & $\mathrm{R}$ & 20 & 2.466 & 0.017 & 149.0 & 0.3 & 0 & \\
\hline $20471+2525$ & BU 364 & 14286 & 2006.784 & $\mathrm{R}$ & 20 & 0.792 & 0.014 & 68.4 & 0.6 & 0 & \\
\hline $20474+3629$ & STT 413Aa-B & 14296 & 2006.784 & $\mathrm{R}$ & 20 & 0.876 & 0.008 & $5.7^{*}$ & 0.5 & 1 & \\
\hline $20531+2909$ & STT 417AB & 14397 & 2006.790 & $\mathrm{R}$ & 20 & 0.880 & 0.015 & 28.2 & 0.7 & 0 & \\
\hline $20553+4231$ & STT 423 & 14432 & 2006.918 & $\mathrm{R}$ & 20 & 2.766 & 0.014 & $76.1^{*}$ & 0.3 & 0 & \\
\hline $20557+0432$ & STF 2735 & 14430 & 2006.820 & $\mathrm{R}$ & 20 & 2.032 & 0.017 & $281.5^{*}$ & 0.3 & 0 & \\
\hline $20577+5849$ & A $756 \mathrm{AB}$ & 14493 & 2006.918 & $\mathrm{R}$ & 20 & 0.565 & 0.018 & 209.3 & 2.1 & 0 & \\
\hline $20591+0418$ & STF 2737AB & 14499 & 2006.869 & $\mathrm{R}$ & 10 & 0.632 & 0.004 & 284.5 & 0.3 & 1 & \\
\hline $20595+5013$ & BU 68 & 14520 & 2006.918 & $\mathrm{R}$ & 20 & 1.941 & 0.025 & $148.7^{*}$ & 0.3 & 0 & \\
\hline $20598+6152$ & BU 472 & 14540 & 2006.831 & $\mathrm{R}$ & 20 & 0.743 & 0.011 & $13.9^{*}$ & 0.4 & 0 & \\
\hline $21015+6643$ & HU 959 & 14578 & 2006.831 & $\mathrm{R}$ & 20 & 1.296 & 0.012 & $160.6^{*}$ & 0.3 & 0 & \\
\hline $21137+6424$ & H 148 & 14783 & 2006.727 & $\mathrm{R}$ & 10 & 0.229 & 0.004 & 235.7 & 1.6 & 1 & \\
\hline $21186+1134$ & $\mathrm{BU} 163 \mathrm{AB}$ & 14839 & 2006.727 & $\mathrm{R}$ & 20 & 0.757 & 0.008 & 257.8 & 0.8 & 1 & \\
\hline $21237+5518$ & A 1892 & 14945 & 2006.727 & $\mathrm{R}$ & 20 & 0.747 & 0.009 & 348.7 & 0.7 & 0 & \\
\hline $21289+1105$ & STF 2799AB & 15007 & 2006.727 & $\mathrm{R}$ & 20 & 1.854 & 0.009 & 261.9 & 0.6 & 1 & \\
\hline $21308+4752$ & A 769 & 15053 & 2006.943 & $\mathrm{R}$ & 20 & 0.704 & 0.014 & 292.9 & 1.0 & 0 & Diffuse \\
\hline $21355+2427$ & HU 371 & 15115 & 2006.953 & $\mathrm{R}$ & 10 & 0.255 & 0.003 & 314.6 & 0.7 & 1 & \\
\hline $21441+2845$ & STF $2822 \mathrm{AB}$ & 15270 & 2006.784 & $\mathrm{R}$ & 20 & 1.781 & 0.009 & $311.3^{*}$ & 0.3 & 1 & \\
\hline $21454+4356$ & HO 168AB & 15295 & 2006.831 & $\mathrm{R}$ & 20 & 0.855 & 0.017 & 223.4 & 0.6 & 0 & \\
\hline $21480+6920$ & STF 2835 & 15350 & 2006.918 & $\mathrm{~W}$ & 20 & 1.904 & 0.012 & $270.1^{*}$ & 0.6 & 0 & \\
\hline $21555+1053$ & $\mathrm{BU} 75 \mathrm{AB}$ & 15447 & 2006.784 & $\mathrm{R}$ & 20 & 0.906 & 0.008 & 19.9 & 1.0 & 1 & \\
\hline $21557+0715$ & STT 452 & 15452 & 2006.915 & $\mathrm{R}$ & 20 & 0.744 & 0.014 & 178.2 & 0.4 & 0 & \\
\hline $21565+0715$ & STT $453 \mathrm{AB}$ & 15464 & 2006.915 & $\mathrm{R}$ & 20 & 0.717 & 0.012 & 268.1 & 0.5 & 0 & \\
\hline $21581-0329$ & STF 2847 & 15494 & 2006.953 & $\mathrm{R}$ & 20 & 0.731 & 0.017 & 125.8 & 0.9 & 0 & \\
\hline $22009+6250$ & HU 976 & 15558 & 2006.820 & $\mathrm{~W}$ & 20 & 1.609 & 0.008 & 54.9 & 0.3 & 0 & \\
\hline $22070+3605$ & STT $462 \mathrm{AB}$ & 15645 & 2006.831 & $\mathrm{R}$ & 20 & 1.079 & 0.014 & $316.8^{*}$ & 0.5 & 0 & \\
\hline $22086+5917$ & STF $2872 \mathrm{BC}$ & 15670 & 2006.790 & $\mathrm{R}$ & 20 & 0.829 & 0.022 & 298.4 & 0.3 & 1 & \\
\hline $22094+2233$ & STF 2868 & 15673 & 2006.954 & $\mathrm{R}$ & 20 & 1.077 & 0.014 & $352.3^{*}$ & 0.4 & 0 & \\
\hline $22100+2308$ & COU 136 & - & 2006.954 & $\mathrm{R}$ & 20 & 0.485 & 0.012 & $27.7^{*}$ & 0.6 & 1 & \\
\hline $22122+6344$ & STF 2884 & 15742 & 2006.918 & $\mathrm{~W}$ & 20 & 2.118 & 0.015 & $142.6^{*}$ & 0.3 & 0 & \\
\hline $22272+1509$ & STF 2905 & 15950 & 2006.915 & $\mathrm{~W}$ & 20 & 3.326 & 0.017 & $104.5^{*}$ & 0.4 & 0 & Diffuse \\
\hline $22312+5052$ & STF 2918 & 16020 & 2006.915 & $\mathrm{R}$ & 20 & 1.643 & 0.015 & $236.8^{*}$ & 0.5 & 0 & Diffuse \\
\hline $22323+5512$ & AG 283 & 16032 & 2006.831 & $\mathrm{~W}$ & 20 & 2.640 & 0.013 & $332.1^{*}$ & 0.3 & 0 & \\
\hline $22328+2625$ & $\mathrm{HO} 475 \mathrm{AB}$ & 16037 & 2006.951 & $\mathrm{~W}$ & 20 & 1.085 & 0.008 & $126.0^{*}$ & 0.8 & 0 & \\
\hline $22419+2126$ & STF 2934 & 16185 & 2006.951 & $\mathrm{~W}$ & 20 & 1.337 & 0.008 & $57.9^{*}$ & 0.4 & 1 & \\
\hline $22426+4401$ & A $414 \mathrm{AB}$ & 16204 & 2006.951 & $\mathrm{~W}$ & 20 & 1.723 & 0.009 & 14.1 & 0.3 & 0 & Diffuse \\
\hline $22485+5409$ & AG 424 & 16280 & 2006.973 & $\mathrm{R}$ & 20 & 2.266 & 0.013 & $135.5^{*}$ & 0.4 & 0 & \\
\hline $22509+5303$ & BU $1332 \mathrm{AB}$ & 16310 & 2006.918 & $\mathrm{~W}$ & 20 & 1.509 & 0.013 & $128.3^{*}$ & 0.4 & 0 & \\
\hline $22514+6142$ & STF 2950AB & 16317 & 2006.784 & $\mathrm{R}$ & 20 & 1.293 & 0.008 & $278.9^{*}$ & 0.3 & 0 & \\
\hline $22537+4445$ & $\mathrm{BU} 382 \mathrm{AB}$ & 16345 & 2006.869 & $\mathrm{R}$ & 20 & 0.823 & 0.008 & $230.7^{*}$ & 0.3 & 1 & \\
\hline $22557+1547$ & HU 987 & 16373 & 2006.790 & $\mathrm{~W}$ & 20 & 1.110 & 0.008 & $78.9^{*}$ & 0.3 & 1 & \\
\hline $22597+4149$ & HLD 56 & 16435 & 2006.790 & $\mathrm{~W}$ & 20 & 1.158 & 0.008 & $93.1^{*}$ & 0.6 & 0 & \\
\hline $23072+6050$ & BU 180AB & 16518 & 2006.869 & $\mathrm{R}$ & 20 & 0.548 & 0.013 & $136.1^{*}$ & 0.6 & 0 & \\
\hline
\end{tabular}


Table 1 Measurements of visual binaries between July and December 2006 (end).

\begin{tabular}{|c|c|c|c|c|c|c|c|c|c|c|c|}
\hline WDS & Name & ADS & Epoch & Fil. & $\begin{array}{l}\text { Eyep. } \\
\text { (mm) }\end{array}$ & $\begin{array}{c}\rho \\
(\operatorname{arcsec})\end{array}$ & $\begin{array}{c}\sigma_{\rho} \\
(\operatorname{arcsec})\end{array}$ & $\begin{array}{c}\theta \\
\text { (deg.) }\end{array}$ & $\begin{array}{c}\sigma_{\theta} \\
\text { (deg.) }\end{array}$ & Orb. & Notes \\
\hline $23078+6338$ & HU 994 & 16530 & 2006.869 & $\mathrm{R}$ & 10 & 0.200 & 0.004 & 314.9 & 1.3 & 1 & \\
\hline $23079+7523$ & STT 489AB & 16538 & 2006.869 & $\mathrm{R}$ & 20 & 1.094 & 0.019 & $350.8^{*}$ & 0.8 & 1 & \\
\hline $23102+5727$ & $\mathrm{STT} 490 \mathrm{AB}$ & 16560 & 2006.869 & $\mathrm{~W}$ & 20 & 1.200 & 0.008 & $296.5^{*}$ & 0.3 & 0 & \\
\hline $23147+4116$ & A 200 & 16621 & 2006.954 & $\mathrm{R}$ & 20 & 0.652 & 0.014 & 74.7 & 2.2 & 0 & Faint \& diffuse \\
\hline $23292+4042$ & A 1487 & 16785 & 2006.954 & $\mathrm{R}$ & 20 & 1.063 & 0.015 & 158.8 & 0.5 & 0 & \\
\hline $23340+3120$ & BU 720 & 16836 & 2006.975 & $\mathrm{R}$ & 10 & 0.554 & 0.005 & $97.7^{*}$ & 0.4 & 1 & \\
\hline $23375+4426$ & STT 500AB & 16877 & 2006.975 & $\mathrm{R}$ & 10 & 0.463 & 0.003 & $8.4^{*}$ & 0.5 & 1 & \\
\hline $23413+3234$ & $\mathrm{BU} 858 \mathrm{AB}$ & 16928 & 2006.975 & $\mathrm{R}$ & 20 & 0.830 & 0.012 & $224.0^{*}$ & 0.8 & 0 & \\
\hline $23431+1150$ & A 1242 & 16951 & 2006.976 & W & 20 & 0.940 & 0.015 & 333.6 & 1.2 & 1 & \\
\hline $23516+4205$ & STT 510AB & 17050 & 2006.954 & $\mathrm{R}$ & 20 & 0.590 & 0.008 & $120.0^{*}$ & 1.1 & 0 & \\
\hline $23590+5315$ & HLD 59AB & 17141 & 2006.954 & $\mathrm{R}$ & 20 & 1.155 & 0.013 & 12.5 & 0.7 & 0 & \\
\hline
\end{tabular}

Note: In column 9, the exponent ${ }^{*}$ indicates that the position angle $\theta$ could be determined without the $180^{\circ}$ ambiguity.

Note that the case of ADS 11454 is noteworthy: it is a quadruple system that we detected as triple only because the two components $\mathrm{A}$ and $\mathrm{B}$ are presently too close $\left(\rho<0^{\prime \prime} .1\right)$ to be resolved with the $102 \mathrm{~cm}$ Zeiss telescope (see discussion in the next section). In order to provide the maximum accuracy, our measures of STF 2339 refer to the position of the components $\mathrm{C}$ and $\mathrm{D}$ relative to that of the unresolved couple AB. This is different from the convention adopted in the WDS Catalogue of reporting the mean position of C-D relative to $\mathrm{AB}$.

\subsection{Quadrant determination}

As mentioned in Sect. 2, we have used the restricted triple-correlation technique of Aristidi et al. (1997) to try resolving the $180^{\circ}$ ambiguity in the $\theta$ measurements made from the auto-correlation files and determine the quadrant containing the companion. For each observation, we examined the location on the triple-correlation file of the faintest secondary spot, which corresponded to that of the companion. When the signal-to-noise ratio was good enough, we were able to unambiguously determine the location of this spot and thus resolve the $180^{\circ}$ ambiguity. For the couples with the largest separations, we could also see the location of the companion directly on the long integration files. As a result, in Table 1 , we are able to give the non-ambiguous angular separation of 119 out of 176 measurements, i.e. $68 \%$ of the total (marked with an asterisk in Col 9).

Note that we used a different method for WAK $21 \mathrm{CD}$. This pair belongs to the the quadruple system of ADS 11454 consisting of STF2339AB-CD = HU 322AB + WAK $21 \mathrm{CD}$. With our observations we detected the three components $\mathrm{AB}, \mathrm{C}$ and $\mathrm{D}$ (see Table 1 ), but we could not resolve the pair A-B (HU 322AB) that is presently too close $\left(\rho<0^{\prime \prime} .1\right)$ for the $102 \mathrm{~cm}$ Zeiss telescope. To determine the quadrant of WAK 21CD we computed the bispectrum from our data recorded on tape and managed to restore an image of the triple system $\mathrm{AB}, \mathrm{C}$ and $\mathrm{D}$, following the procedure described in Prieur et al. (2003). This image and the mean autocorrelation are displayed in Fig. 2. Our observations thus lead to a position angle of $265.5^{\circ}$ for WAK $21 \mathrm{CD}$. This is in agreement both with the quadrant published in the last version of the WDS Catalogue, and with the speckle measurements of Scardia et al. (2000) and Balega et al. (2002), that are reported in IC4 (both were made in 1998).

When checking whether those "absolute" $\theta$ values were consistent with the values tabulated in IC4, we found a good agreement for all objects, except for ADS 12770,15950 , and 16037 . We now discuss the cases of those three objects, using the usual convention of numbering the quadrants from 1 to 4 to indicate the North-East, South-East, South-West and North-West quadrants, respectively.

ADS 12770: our quadrant determination $(\mathrm{Q}=1)$ seems robust with a clear contrast between the two secondary peaks of the triple correlation. It is nevertheless in contradiction with the third quadrant reported in IC4, relative to observations made in the $V$ band. Our quadrant determination was made without any filter (i.e. in $W$ ), which results in a spectral band close to the $R$ band (see Sect. 3). This may be the origin of the discrepancy, and a quadrant inversion could occur between $V$ and $R$, since the two stars have a small magnitude difference: $\Delta m_{V}=0.1$ mag. according to Germain et al. (1999).

ADS 15950: here also, our quadrant determination $(\mathrm{Q}=2)$ seems reliable and was made without any filter, whereas the quadrant of $\mathrm{Q}=4$ reported in $\mathrm{IC} 4$ refers to the $V$ band. The difference of magnitude is even smaller than for ADS 12770, with $\Delta m_{V}=0.06$ mag. (WDS), 


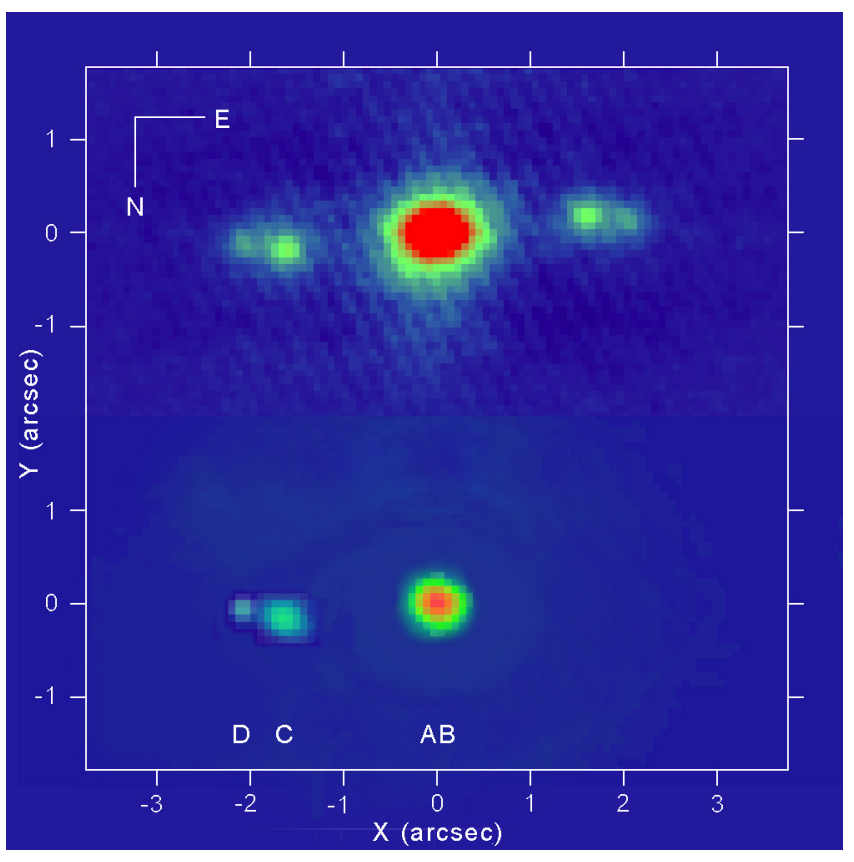

Fig. 2 Triple system (AB, C, D) of ADS 11454: autocorrelation (top) and restored image with bispectral methods (bottom). To improve the clarity of this image, we have subtracted a circular halo that existed around the central component after deconvolution, and whose origin was due to an imperfect matching of the seeing between the object and the reference star.

which could naturally explain a quadrant inversion between $V$ and $R$.

ADS 16037: in this case, our quadrant determination $(\mathrm{Q}=2)$ is not as clear as the others, but the signalto-noise ratio is still satisfactory. IC4 reports a quadrant $\mathrm{Q}=4$ in disagreement with our determination that was also made without any filter (in $W$ ) because of the low luminosity of this object. The magnitude difference is slightly larger in this case, with $\Delta m_{V} \approx 0.25 \mathrm{mag}$ and an integrated spectral type F5. Hence a quadrant inversion is less likely, but cannot be excluded. We intend to re-observe this pair as soon as possible, as well as ADS 12770 and 15950, to check our quadrant determinations.

Finally, there is a quadrant discrepancy for ADS 12201 and 14839, when considering the quadrant convention adopted for the orbit computation by some authors (see next Section). Their convention seems doubtful since it is in contradiction with the last observations reported in IC4.

\subsection{Comparison with published ephemerides}

The $(O-C)$ (Observed minus Computed) residuals of the measurements for the 58 systems with a known orbit of Table 1 are displayed in Table 2 in Cols. 6 and 7 for the separation $\rho$ and position angle $\theta$, respectively. The orbital elements used for computing the ephemerides were retrieved from OC6 and from our last publications (Scardia et al. 2006, 2008a, 2008b). When extracted from OC6, the corresponding bibliographic references are indicated in Col. 3 as they appear in this catalogue, with an asterisk. The $\rho$ values in Col. 5 are the relevant observed separations, taken from Col. 7 of Table 1 . They are repeated here for the convenience of the reader, to be able to identify the cases when $\rho$ is small. For ADS 11479, 11584 and 16538, we also give the residuals obtained with our new orbits presented in Sect. 4, for comparison.

We noted a $180^{\circ}$ discrepancy of the position angle between our measurements and the published ephemerides for ADS 12201 and 14839 (see Sect. 3.1). This is indicated with the superscript ${ }^{Q}$ in Col. 7 .

The residuals reported in Table 2 were computed with the most recent orbits found in OC6, but for some objects, we also give the $O-C$ values relative to old orbits found in the previous issues of OC6, when they are still valid. This includes ADS 11635AB, 12889AB, 15115,16373 for which the "old" and "new" orbits lead to comparable residuals. For ADS 11956, we used our last orbit published in Scardia et al. (2008b), that was computed using the PISCO measurement reported in Table 1.

Fig. 3 shows that the residuals are well centered around the origin, with a rather large scatter that can be explained by the (old) age of many orbits. The average values computed with the 63 residuals of Table 2 are $\left\langle\Delta \rho_{O-C}\right\rangle=0^{\prime \prime} .00 \pm 0^{\prime \prime} .09$ and $\left\langle\Delta \theta_{O-C}\right\rangle=$ $-0^{\circ} .3 \pm 1^{\circ} .8$. The small values obtained for those offsets provide a new validation of our calibration made with a grating mask (see Paper III), which is thus in good agreement with the measurements made by the other observers.

A very large residual in separation was computed for ADS 13256 with Hopmann (1973)'s orbit: $\Delta \rho_{O-C}=$ $-0^{\prime \prime} .54$. Unfortunately, the motion seems rectilinear for this object. The arc of orbit is very short and the derivation of valid orbital parameters is still impossible.

Concerning the position angles, the largest absolute values of the residuals were found for ADS 11584, with $\Delta \theta=-6.1^{\circ}$ (Alzner 2006), and ADS 16538, with $\Delta \theta=$ $-5.3^{\circ}$ (Baize 1992). We propose new orbital elements for those objects in the next section.

\section{Revised orbits for ADS 11479, 11584 and 16538}

In this section we present the new orbits we have computed for ADS 11584 and 16538, for which the previously published orbits resulted in large residuals from our measurements. We also computed new orbital elements for ADS 11479 since its orbit has not been revised for more than forty years although numerous observations have been done since. 
Table 2 Residuals of the measurements of Table 1 with published orbits (begin.). In col. 3, an asterisk indicates that the references are reported as they appear in the Sixth Catalog of Orbits of Hartkopf \& Mason (2008). The other references can be found in Sect. References, at the end of this paper. In col. $7,{ }^{Q}$ indicates discrepant quadrants between our measurements and those orbits.

\begin{tabular}{|c|c|c|c|c|c|c|}
\hline$\overline{\mathrm{ADS}}$ & Name & Orbit & Epoch & $\begin{array}{c}\rho(\mathrm{O}) \\
(\operatorname{arcsec}) \\
\end{array}$ & $\begin{array}{r}\Delta \rho(\mathrm{O}-\mathrm{C}) \\
(\operatorname{arcsec}) \\
\end{array}$ & $\begin{array}{r}\Delta \theta(\mathrm{O}-\mathrm{C}) \\
(\text { deg. }) \\
\end{array}$ \\
\hline 10157 & STF 2084 & Söderhjelm (1999)* & 2006.642 & 1.019 & -0.03 & 0.3 \\
\hline 10235 & STF $2107 \mathrm{AB}$ & Scardia et al. $(2003 \mathrm{c})^{*}$ & 2006.680 & 1.452 & 0.07 & -1.3 \\
\hline 10279 & STF 2118 AB & Scardia et al. $(2002 d)^{*}$ & 2006.639 & 1.067 & -0.09 & -1.3 \\
\hline 10345 & STF $2130 \mathrm{AB}$ & Heintz $(1981 b)^{*}$ & 2006.639 & 2.360 & 0.05 & 1.9 \\
\hline 10531 & HU 1179 & Hartkopf (2000b)* & 2006.675 & 0.247 & -0.01 & 1.7 \\
\hline 10598 & STF 2173 & Heintz $(1994 a)^{*}$ & 2006.680 & 0.451 & -0.01 & 0.5 \\
\hline 10699 & STF 2199 & Popovic \& Pavlovic (1995d)* & 2006.694 & 2.007 & 0.09 & 1.4 \\
\hline 10743 & HU 1285 & Seymour et al. $(2002)^{*}$ & 2006.677 & 0.546 & 0.01 & -0.2 \\
\hline 10769 & STF 2205 & Cvetkovic $(2007 a)^{*}$ & 2006.696 & 1.076 & -0.03 & -0.4 \\
\hline 10795 & STF 2215 & Cvetkovic \& Novakovic $(2006 \mathrm{e})^{*}$ & 2006.677 & 0.505 & 0.02 & 1.2 \\
\hline 10912 & STF 2244 & Heintz $(1997)^{*}$ & 2006.680 & 0.611 & 0.08 & -1.5 \\
\hline 11010 & BU 1127 Aa-B & Cvetkovic \& Novakovic $(2006 \mathrm{e})^{*}$ & 2006.639 & 0.775 & -0.05 & -1.5 \\
\hline 11111 & STF $2281 \mathrm{AB}$ & Söderhjelm $(1999)^{*}$ & 2006.718 & 0.602 & 0.00 & 1.4 \\
\hline$"$ & $"$ & $"$ & 2006.721 & 0.588 & -0.01 & 1.2 \\
\hline 11123 & STF 2289 & Hopmann $(1964 b)^{*}$ & 2006.694 & 1.231 & -0.01 & 2.3 \\
\hline 11128 & HU 674 & Seymour et al. $(2002)^{*}$ & 2006.677 & 0.753 & 0.12 & 0.2 \\
\hline 11186 & STF 2294 & Luyten $(1934 a)^{*}$ & 2006.696 & 1.295 & 0.10 & -0.7 \\
\hline 11311 & STT $353 \mathrm{AB}$ & Andrade $(2005)^{*}$ & 2006.699 & 0.480 & 0.00 & -0.9 \\
\hline 11324 & $\mathrm{AC} 11$ & Heintz $(1995)^{*}$ & 2006.696 & 0.873 & 0.03 & -0.1 \\
\hline 11334 & STF 2315 AB & Mason et al. $(2004 b)^{*}$ & 2006.696 & 0.642 & 0.01 & 0.7 \\
\hline 11339 & BU 1203 & Popovic \& Pavlovic (1996b)* & 2006.726 & 0.459 & -0.01 & -0.6 \\
\hline 11468 & A $1377 \mathrm{AB}$ & Scardia $(1984 \mathrm{e})^{*}$ & 2006.677 & 0.251 & 0.01 & -4.1 \\
\hline 11479 & STT 359 & Symms (1964) & 2006.639 & 0.729 & 0.01 & 0.7 \\
\hline$"$ & $"$ & This paper & 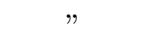 & $"$ & -0.00 & 0.1 \\
\hline 11483 & STT 358 AB & Heintz $(1995)^{*}$ & 2006.675 & 1.655 & 0.10 & 0.8 \\
\hline 11579 & STF 2367 AB & Pourbaix $(2000 \mathrm{~b})^{*}$ & 2006.743 & 0.378 & 0.00 & -0.2 \\
\hline 11584 & STT 363 & Alzner (2006) & 2006.727 & 0.431 & 0.01 & -6.1 \\
\hline$"$ & $"$ & This paper & $"$ & $"$ & 0.02 & -2.9 \\
\hline 11635 & STF 2382 AB & Mason et al. $(2004 b)^{*}$ & 2006.675 & 2.380 & -0.03 & 0.3 \\
\hline & & Novakovic \& Todorovic $(2006 \mathrm{e})^{*}$ & 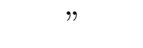 & $"$ & 0.00 & 0.2 \\
\hline 11635 & STF 2383 Cc-D & Docobo \& Costa $(1984 b)^{*}$ & 2006.675 & 2.381 & 0.02 & -0.1 \\
\hline 11871 & BU $648 \mathrm{AB}$ & Heintz $(1994 a)^{*}$ & 2006.721 & 0.898 & -0.03 & 1.5 \\
\hline 11897 & STF 2438 & Hartkopf \& Mason (2001a)* & 2006.716 & 0.867 & 0.03 & 0.9 \\
\hline 11956 & STF 2437 & Scardia et al. (2008b) & 2006.743 & 0.580 & 0.00 & 0.1 \\
\hline 12040 & STF 2454 AB & Starikova $(1982 \mathrm{~b})^{*}$ & 2006.822 & 1.326 & 0.00 & 0.5 \\
\hline 12201 & STF 2484 & Hopmann $(1973 b)^{*}$ & 2006.694 & 2.163 & -0.12 & $0.3^{Q}$ \\
\hline 12447 & STF 2525 & Heintz $(1984 b)^{*}$ & 2006.677 & 2.130 & 0.05 & -0.5 \\
\hline 12540 & MCA 55 Aac & Scardia et al. (2008a) & 2006.721 & 0.359 & -0.02 & -2.5 \\
\hline 12880 & STF 2579 AB & Scardia $(1983 a)^{*}$ & 2006.721 & 2.666 & 0.01 & -1.0 \\
\hline$"$ & $"$ & Scardia $(1983 a)^{*}$ & 2006.762 & 2.647 & -0.01 & -0.9 \\
\hline 12889 & STF 2576 AB & Scardia $(1981)^{*}$ & 2006.699 & 2.832 & -0.04 & -0.9 \\
\hline & $"$ & Söderhjelm (1999)* & $"$ & $"$ & -0.01 & -0.3 \\
\hline 12972 & STT 387 & Mason et al. $(2006 \mathrm{~b})^{*}$ & 2006.724 & 0.571 & 0.00 & 0.1 \\
\hline 13256 & STF 2613 & Hopmann (1973) & 2006.727 & 3.637 & -0.54 & 2.3 \\
\hline 13461 & STT 400 & Heintz $(1997)^{*}$ & 2006.699 & 0.593 & 0.01 & 0.1 \\
\hline 14073 & $\mathrm{BU} 151 \mathrm{AB}$ & Alzner $(1998 \mathrm{a})^{*}$ & 2006.790 & 0.532 & -0.01 & -0.6 \\
\hline 14296 & STT 413 Aa-B & Rabe $(1948 b)^{*}$ & 2006.784 & 0.876 & -0.03 & 2.7 \\
\hline 14499 & STF 2737 AB & Zeller $(1965)^{*}$ & 2006.869 & 0.632 & 0.01 & 0.5 \\
\hline 14783 & H1 48 & Scardia et al. (2008a) & 2006.727 & 0.229 & -0.06 & -3.1 \\
\hline 14839 & $\mathrm{BU} 163 \mathrm{AB}$ & Fekel et al. $(1997)^{*}$ & 2006.727 & 0.757 & 0.02 & $-1.5^{Q}$ \\
\hline 15007 & STF 2799 AB & Popovic $(1987)^{*}$ & 2006.727 & 1.854 & 0.12 & 2.1 \\
\hline 15115 & HU 371 & Scardia et al. (2006) & 2006.953 & 0.255 & -0.05 & 0.9 \\
\hline & $"$ & Mason et al. $(2006 \mathrm{~b})^{*}$ & $"$ & ${ }^{2}>$ & -0.03 & -1.1 \\
\hline 15270 & STF $2822 \mathrm{AB}$ & Heintz $(1995)^{*}$ & 2006.784 & 1.781 & 0.06 & -2.3 \\
\hline 15447 & $\mathrm{BU} 75 \mathrm{AB}$ & Heintz $(1996 \mathrm{a})^{*}$ & 2006.784 & 0.906 & -0.02 & -0.8 \\
\hline
\end{tabular}


Table 2 Residuals of the measurements of Table 1 with published orbits (end).

\begin{tabular}{|c|c|c|c|c|c|c|}
\hline$\overline{\mathrm{ADS}}$ & Name & Orbit & Epoch & $\begin{array}{c}\rho(\mathrm{O}) \\
(\operatorname{arcsec})\end{array}$ & $\begin{array}{r}\Delta \rho(\mathrm{O}-\mathrm{C}) \\
(\operatorname{arcsec})\end{array}$ & $\begin{array}{r}\Delta \theta(\mathrm{O}-\mathrm{C}) \\
\text { (deg.) }\end{array}$ \\
\hline 15670 & STF $2872 \mathrm{BC}$ & Seymour et al. $(2002)^{*}$ & 2006.790 & 0.829 & 0.01 & -0.2 \\
\hline- & COU 136 & Couteau $(1999 b)^{*}$ & 2006.954 & 0.485 & -0.03 & 2.4 \\
\hline 16185 & STF 2934 & Heintz $(1981 \mathrm{a})^{*}$ & 2006.951 & 1.337 & 0.14 & -0.5 \\
\hline 16345 & BU 382 AB & Söderhjelm (1999)* & 2006.869 & 0.823 & -0.04 & 0.8 \\
\hline 16373 & HU 987 & Heintz $(1984 a)^{*}$ & 2006.790 & 1.110 & 0.14 & -0.5 \\
\hline$"$ & $"$ & Brendley $(2007 a)^{*}$ & $"$ & $"$ & 0.05 & -1.3 \\
\hline 16530 & HU 994 & Docobo $(1991 \mathrm{e})^{*}$ & 2006.869 & 0.200 & -0.05 & 2.6 \\
\hline 16538 & STT 489 AB & Baize (1992) & 2006.869 & 1.094 & -0.09 & -5.3 \\
\hline$"$ & $"$ & This paper & $"$ & $"$ & -0.02 & -2.1 \\
\hline 16836 & BU 720 & Starikova $(1982 \mathrm{~b})^{*}$ & 2006.975 & 0.554 & 0.02 & -3.8 \\
\hline 16877 & STT $500 \mathrm{AB}$ & Zulevic $(1981)^{*}$ & 2006.975 & 0.463 & 0.03 & -4.2 \\
\hline 16951 & A 1242 & Ling $(2004 c)^{*}$ & 2006.976 & 0.940 & 0.00 & -2.5 \\
\hline
\end{tabular}

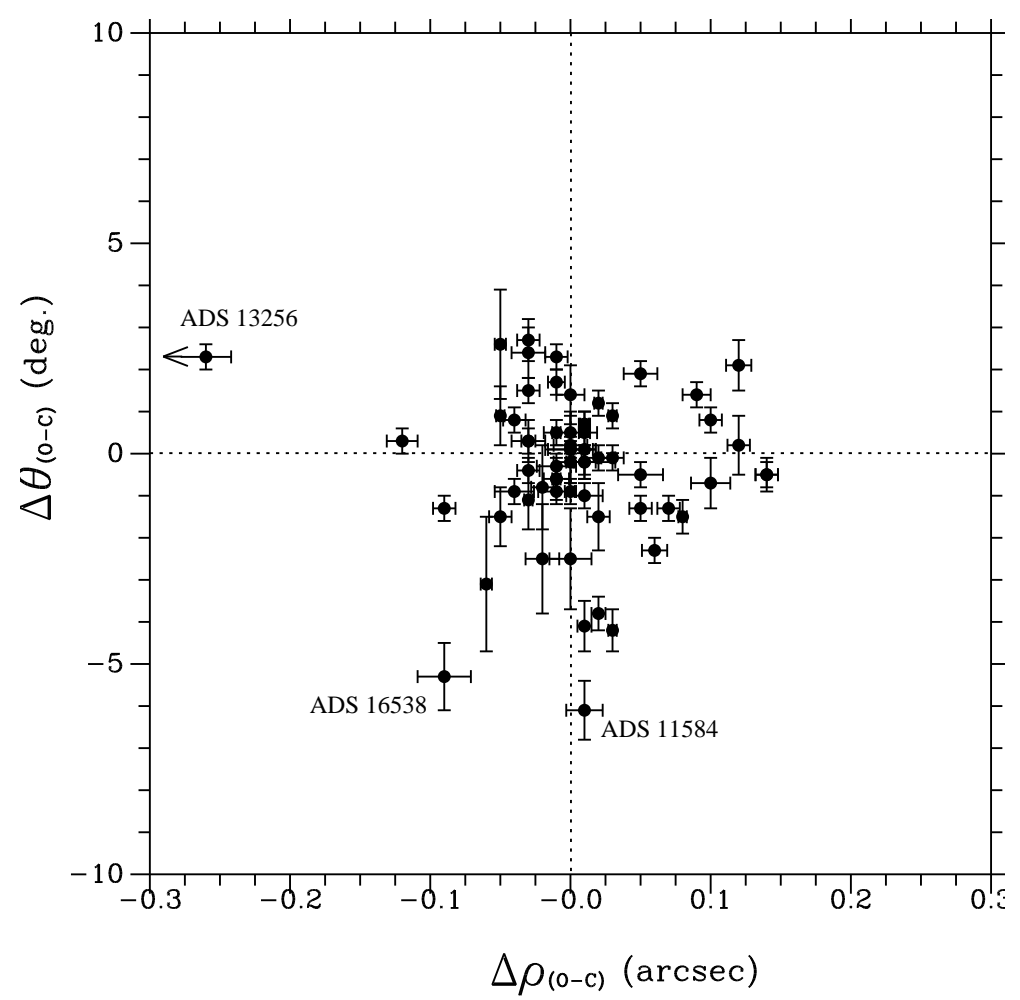

Fig. 3 Residuals of our measurements from the published orbits. The data point of the residual of ADS 13256, computed with Hopmann (1973)'s orbit, lies outside of this frame.

We have followed the same method for the three objects, using our last measurements with PISCO and the other available observations contained in the data base maintained by the United States Naval Observatory. We first computed the preliminary orbital elements with the analytical method of Kowalsky (1873), and then used them as initial values for the least-squares method of Hellerich (1925).

The final orbital elements are presented in Table 3. In this table, $\Omega_{2000}$ is the position angle of the ascending node, measured in the plane of the sky from north through east, taking the equinox of 2000 as a reference, $\omega$ is the longitude of the periastron in the plane of the true orbit, measured from the ascending node to the periastron, in the direction of motion of the companion, $i$ is the inclination of the orbit relative to the plane of the sky, $e$ the eccentricity, $T$ the epoch of periastron passage, $P$ the period, $n$ the mean angular motion, and $a$ is the semi-major axis. The four parameters A, B, F, and $\mathrm{G}$ are the Thiele-Innes constants (useful for an easier computation of the ephemerides). 
The corresponding $(O-C)$ residuals, restricted to the last observations for reasons of space, are given in Tables 4, 5 and 6 for ADS 11479, 11584 and 16538, respectively. For each measurement, the date in Besselian years is given in Col. 1 , and the $(O-C)$ residuals in $\rho$ and $\theta$ in Cols. 2 and 3, respectively.

The apparent orbits are shown in Fig. 4 as solid lines and the observational data used for the calculation of the orbital elements are plotted as small crosses. The orientation of the graphs conforms to the convention adopted by the observers of visual binary stars. For each object, the big cross indicates the location of the primary component, and the straight line going through this point is the line of apsides. The sense of rotation of the companion is indicated with an arrow.

The ephemerides for 2008-2015 are presented in Table 7, with the date in Besselian years in Col. 1, the angular separations $\rho$ in Cols. 2, 4 and 6, and the position angles $\theta$ in Cols. 3, 5 and 7 .

We present some physical parameters derived for those systems from our orbital elements in Table 8. For each object whose name is given in Col. 1 we report its spectral type in Col. 2, the Hipparcos parallax $\pi_{H I P}$ in Col. 3, the apparent angular extent of the semi major axis $a$ in arcseconds in Col. 4 (from Table 3), the linear size of $a$ in AU in Col. 5, and the total mass of the system $\mathfrak{M}_{\text {total }}$ in Col. 6 . Both the linear size of $a$ and $\mathfrak{M}_{\text {total }}$ were computed from our orbital elements and using the Hipparcos parallax.

\subsection{New orbit of ADS 11479 - STT 359}

The revision of the orbit of ADS 11479 is mainly justified because the previous orbit is very old (Symms 1964) and we have taken advantage of the numerous measurements obtained by speckle interferometry. Indeed, out of the 257 measurements now available for this object, 132 were obtained since 1964, mostly with speckle techniques. The new orbital elements reported in Table 3 were computed with the analytical method of Kowalsky (1873) followed by the least-squares method of Hellerich (1925). During this computation, it appeared that 13 measurements had to be rejected because they were clearly aberrant. The standard deviations of the residuals are $0^{\prime \prime} .05$ and $2^{\circ} .0$ for $\rho$ and $\theta$, respectively.

The total mass of the system derived from our orbit is $5.3 \pm 3.3 \mathrm{M}_{\odot}$ ( (see Table 8 ), which is in good agreement with the value expected for a system of this spectral type (G9 III).

\subsection{New orbit of ADS 11584 - STT 363}

The orbital elements reported in Table 3 were derived from the 58 available measurements obtained since 1843. The standard deviations of the residuals are $0^{\prime \prime} .05$ and $4^{\circ} .7$ for $\rho$ and $\theta$, respectively.
Table 4 ADS 11479: O-C residuals of our new orbit (after 1995). The symbol ${ }^{P}$ indicates PISCO measurements.

\begin{tabular}{ccc}
\hline Epoch & $\begin{array}{r}\Delta \rho(\mathrm{O}-\mathrm{C}) \\
(\operatorname{arcsec})\end{array}$ & $\begin{array}{r}\Delta \theta(\mathrm{O}-\mathrm{C}) \\
(\text { deg. })\end{array}$ \\
\hline 1997.537 & -0.023 & 0.6 \\
1997.537 & -0.023 & 0.4 \\
1997.579 & -0.013 & 0.1 \\
1997.601 & -0.003 & 0.0 \\
1997.609 & -0.023 & -0.5 \\
1997.658 & -0.033 & -0.6 \\
1997.669 & -0.003 & 0.0 \\
1998.673 & $0.004^{P}$ & $0.7^{P}$ \\
1999.512 & -0.020 & -1.7 \\
1999.512 & -0.030 & -1.3 \\
1999.550 & 0.030 & 0.2 \\
1999.728 & 0.001 & 1.0 \\
1999.745 & -0.010 & 0.4 \\
2000.546 & -0.023 & 0.2 \\
2001.417 & -0.025 & 0.5 \\
2001.580 & 0.024 & 1.1 \\
2002.460 & -0.059 & 0.4 \\
2002.510 & 0.011 & 1.7 \\
2003.598 & -0.002 & 0.9 \\
2004.369 & 0.016 & 1.1 \\
2004.640 & $-0.003^{P}$ & $0.5^{P}$ \\
2004.694 & 0.015 & 0.2 \\
2006.389 & -0.009 & 0.4 \\
2006.639 & $-0.001^{P}$ & $0.1^{P}$ \\
\hline
\end{tabular}

Table 5 ADS 11584: O-C residuals of our new orbit (after 1995). The symbol ${ }^{P}$ indicates PISCO measurements.

\begin{tabular}{crr}
\hline Epoch & $\begin{array}{r}\Delta \rho(\mathrm{O}-\mathrm{C}) \\
(\text { arcsec })\end{array}$ & $\begin{array}{r}\Delta \theta(\mathrm{O}-\mathrm{C}) \\
(\text { deg. })\end{array}$ \\
\hline 1996.430 & 0.025 & 4.5 \\
1996.600 & 0.051 & 6.0 \\
1997.540 & 0.081 & 9.6 \\
1999.570 & 0.030 & 8.8 \\
2000.610 & 0.040 & 7.2 \\
2001.470 & 0.025 & 3.8 \\
2002.540 & 0.046 & 7.4 \\
2002.581 & 0.016 & -0.8 \\
2003.560 & 0.019 & 2.1 \\
2004.510 & 0.014 & 3.6 \\
2005.630 & 0.007 & 0.6 \\
2006.727 & $0.022^{P}$ & $-2.9^{P}$ \\
\hline
\end{tabular}

In the last 35 years, the best orbits have been calculated by Zulevic (1975), Baize (1989) and Alzner (1996), with increasing values for the orbital period. Alzner (2006)'s orbit leads to a large residual with our last measurement: $\Delta \theta_{O-C}=-6^{\circ} .1$. As the declination of $\mathrm{ADS} 11584$ is high (close to $77^{\circ}$ ), all the measurements had to be carefully corrected for precession effects (which reached $4^{\circ}$ during the 160 year time-range of the observations). As the angular sepa- 
Table 3 New orbital elements of ADS 11479, 11584 and 16538.

\begin{tabular}{|c|c|c|c|c|c|c|c|c|c|c|c|c|}
\hline$\overline{\mathrm{ADS}}$ & $\begin{array}{l}\Omega_{2000} \\
\text { (deg.) }\end{array}$ & $\begin{array}{c}\omega \\
\text { (deg.) }\end{array}$ & $\begin{array}{c}i \\
\text { (deg.) }\end{array}$ & $e$ & $\begin{array}{c}T \\
(\mathrm{yr}) \\
\end{array}$ & $\begin{array}{c}P \\
(\mathrm{yr}) \\
\end{array}$ & $\begin{array}{c}n \\
\text { (deg./yr) }\end{array}$ & $\begin{array}{c}a \\
(\operatorname{arcsec}) \\
\end{array}$ & $\begin{array}{c}\mathrm{A} \\
(\operatorname{arcsec})\end{array}$ & $\begin{array}{c}\mathrm{B} \\
(\operatorname{arcsec})\end{array}$ & $\begin{array}{c}\mathrm{F} \\
(\operatorname{arcsec})\end{array}$ & $\begin{array}{c}\mathrm{G} \\
(\operatorname{arcsec})\end{array}$ \\
\hline 11479 & $\begin{array}{l}10.0 \\
\pm 1.2\end{array}$ & $\begin{array}{l}197.7 \\
\pm 2.1\end{array}$ & $\begin{array}{l}119.9 \\
\pm 2.0\end{array}$ & & $\begin{array}{c}1929.081 \\
\pm 0.54\end{array}$ & $\begin{array}{l}219.3 \\
\pm 8.8\end{array}$ & & $\begin{array}{c}0.425 \\
\pm 0.014\end{array}$ & -0.40992 & -0.00687 & 0.09220 & 0.22120 \\
\hline 1 & $\begin{array}{l}159.7 \\
\pm 1.5\end{array}$ & $\begin{array}{l}55.9 \\
\pm 4.8\end{array}$ & $\begin{array}{l}78.3 \\
\pm 1.7\end{array}$ & $\begin{array}{c}0.890 \\
\pm 0.057\end{array}$ & $\begin{array}{c}1987.038 \\
\pm 0.72\end{array}$ & $\begin{array}{c}641.9 \\
\pm 506.0\end{array}$ & $\begin{array}{c}0.56083 \\
\pm 0.44\end{array}$ & $\begin{array}{c}0.950 \\
\pm 0.244\end{array}$ & 37 & 16 & 033 & 22 \\
\hline 16538 & $\begin{array}{l}90.3 \\
\pm 4.9\end{array}$ & $\begin{array}{l}90.0 \\
\pm 4.4\end{array}$ & $\begin{array}{l}30.0 \\
\pm 3.0\end{array}$ & $\begin{array}{c}0.5968 \\
\pm 0.0067\end{array}$ & $\begin{array}{c}1934.573 \\
\pm 0.35\end{array}$ & $\begin{array}{l}162.8 \\
\pm 2.8\end{array}$ & $\begin{array}{r}2.21147 \\
\pm 0.038\end{array}$ & $\begin{array}{c}0.810 \\
\pm 0.050\end{array}$ & 147 & 0.00367 & 0.00424 & -0.80999 \\
\hline
\end{tabular}
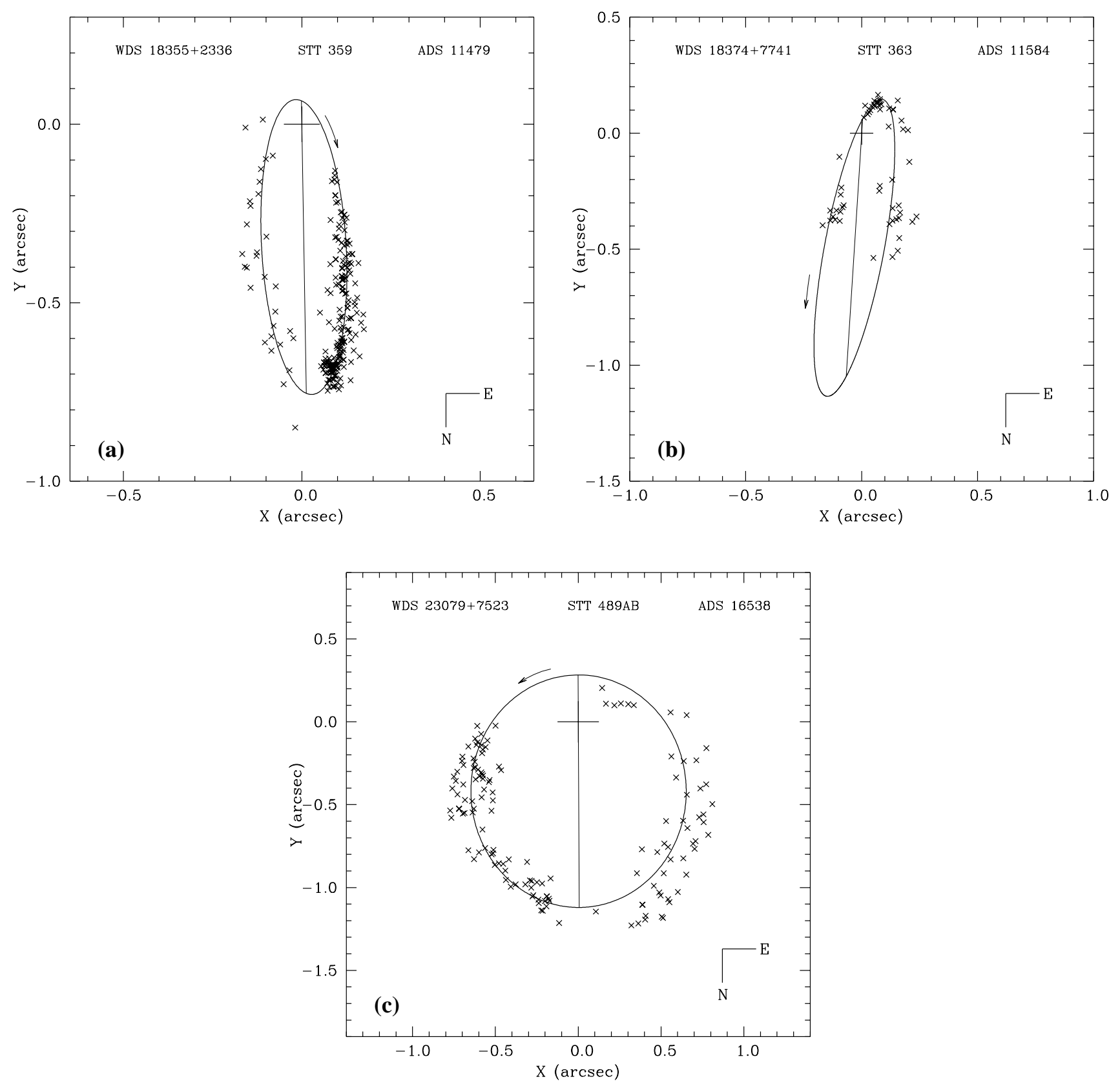

Fig. 4 New orbits of ADS 11479 (a), ADS 11584 (b) and ADS 16538 (c). 
Table 6 ADS 16538: O-C residuals of our new orbit (after 1995). The symbol ${ }^{P}$ indicates PISCO measurements.

\begin{tabular}{crr}
\hline Epoch & $\begin{array}{r}\Delta \rho(\mathrm{O}-\mathrm{C}) \\
(\operatorname{arcsec})\end{array}$ & $\begin{array}{r}\Delta \theta(\mathrm{O}-\mathrm{C}) \\
(\text { deg. })\end{array}$ \\
\hline 1996.760 & -0.045 & -0.4 \\
1996.940 & 0.075 & 4.2 \\
1997.660 & -0.088 & -1.6 \\
1998.770 & -0.022 & 3.5 \\
1998.774 & -0.011 & -0.8 \\
1998.790 & -0.002 & -1.0 \\
1999.690 & -0.095 & 0.4 \\
2000.690 & 0.022 & -0.2 \\
2002.770 & -0.034 & 0.2 \\
2004.974 & $-0.027^{P}$ & $-0.9^{P}$ \\
2005.750 & 0.020 & -1.8 \\
2006.869 & $-0.018^{P}$ & $-2.1^{P}$ \\
\hline
\end{tabular}

Table 7 New ephemerides of ADS 11479, 11584 and 16538.

\begin{tabular}{ccccccc}
\hline \multirow{2}{*}{ Epoch } & \multicolumn{2}{c}{ ADS 11479 } & \multicolumn{2}{c}{ ADS 11584} & \multicolumn{2}{c}{ ADS 16538} \\
& $\begin{array}{c}\theta \\
\text { (arcsec) }\end{array}$ & $\begin{array}{c}\rho \\
\text { (deg.) }\end{array}$ & $\begin{array}{c}\theta \\
\text { (arcsec) }\end{array}$ & $\begin{array}{c}\rho \\
\text { (deg.) }\end{array}$ & $\begin{array}{c}\theta \\
\text { (arcsec) }\end{array}$ & (deg.) \\
\hline 2008.0 & 0.733 & 5.3 & 0.427 & 340.1 & 1.114 & 353.9 \\
2009.0 & 0.735 & 5.2 & 0.441 & 340.4 & 1.116 & 354.7 \\
2010.0 & 0.737 & 5.0 & 0.454 & 340.6 & 1.117 & 355.5 \\
2011.0 & 0.739 & 4.9 & 0.467 & 340.8 & 1.118 & 356.3 \\
2012.0 & 0.741 & 4.7 & 0.480 & 340.9 & 1.119 & 357.1 \\
2013.0 & 0.743 & 4.6 & 0.492 & 341.1 & 1.119 & 357.9 \\
2014.0 & 0.744 & 4.5 & 0.504 & 341.3 & 1.120 & 358.7 \\
2015.0 & 0.746 & 4.3 & 0.516 & 341.4 & 1.120 & 359.5 \\
\hline
\end{tabular}

Table 8 Physical parameters ( $a$ and $\left.\mathfrak{M}_{\text {total }}\right)$ derived from the new orbital elements.

\begin{tabular}{ccrrrr}
\hline Name & Sp. type & $\pi_{H I P}$ & \multicolumn{2}{c}{$a$} & $\mathfrak{M}_{\text {total }}$ \\
& & $(\mathrm{mas})$ & $\left({ }^{\prime \prime}\right)$ & $(\mathrm{AU})$ & $\mathrm{M}_{\odot}$ \\
\hline ADS 11479 & G9 III & 6.70 & 0.425 & 63 & 5.3 \\
& & \pm 1.38 & \pm 0.014 & \pm 13 & \pm 3.3 \\
ADS 11584 & \multirow{2}{*}{ F0 IV } & 7.94 & 0.95 & 120 & 4.2 \\
& & \pm 0.51 & \pm 0.24 & \pm 31 & \pm 7.3 \\
ADS 16538 & \multirow{2}{*}{ G2 III } & 13.8 & 0.81 & 59 & 7.6 \\
& & \pm 0.4 & \pm 0.05 & \pm 4 & \pm 1.6 \\
\hline
\end{tabular}

ration is smaller than $1^{\prime \prime}$, this couple is difficult to observe and the measurements are very scattered. Some of them are clearly aberrant. Even the measurement of 1991.25 made by Hipparcos $\left(\rho=0^{\prime \prime} .14, \theta=316^{\circ} .8\right)$ is doubtful. Nearly all the most recent observations are from Alzner, but the angular position measurements are rather scattered.

Convergence was difficult to obtain, and the weighting scheme appeared crucial in the process. This explains why our orbit is rather different from Alzner's, with a longer period ( $P=642 \mathrm{yr}$ instead of $P=405 \mathrm{yr}$ for Alzner's orbit) and a larger eccentricity. The total mass derived from our elements of $4.2 \mathrm{M}_{\odot}$ (Table 8 ) is in agreement with the expected value for a system of this spectral type (F0 IV). Unfortunately this result is affected by a very large uncertainty $\left( \pm 7.3 \mathrm{M}_{\odot}\right.$ !), that results from the large uncertainty of the period and of the major axis, due to an incomplete coverage of the orbit (see Fig. 4b). Using the formula that we have obtained for luminosity class IV in Scardia et al. (2008b), we find a dynamical parallax of $\pi_{\mathrm{dyn}}=8.50 \mathrm{mas}$, in good agreement with the Hipparcos measurement.

Even though we have obtained convergence on the orbital elements and thus derived their uncertainties, and although the systemic mass is in agreement which the expected mass for a system of this spectral type, the orbit of ADS 11584 should still be considered as badly determined, especially its period and its semimajor axis.

\subsection{New orbit of ADS 16538 - STT 489 AB}

$\pi$ Cephei (HR 8819, HD 218658, ADS 16538) is a multiple system, whose main system A-B is constituted of a bright star with a faint companion. The resulting large magnitude difference makes measurements difficult to perform. It was first discovered as a visual binary by Otto Struve in 1843 (hence the name STT 489 AB for the visual binary), and later the primary star (G2 III according to SIMBAD whereas the WDS reports G0 IV) was found to be a spectroscopic binary with the Lick $0.91 \mathrm{~m}$ refractor by Campbell (1901). The first spectroscopic orbit of the Aa-Ab system was determined by Harper (1925) and recently improved by Scarfe et al. (1983).

The orbit of STT 489 AB could have been well constrained by the measurements made during the periastron passage of 1934. Unfortunately, the observations were difficult to perform at that time (with $\theta \approx 0^{\prime \prime} .3$ ) and perturbed by World War II, at least in Europe where very few astronomers could observe. The most recent orbit was computed by Baize (1992) with the measurements made until 1989. The last measurements lead now to negative residuals in $\rho$ and $\theta$. Note that Baize did not report any equinox value, because he probably did not perform any correction for precession effects.

From the 155 measurements obtained since 1846, we derived the orbital elements of STT 489 AB presented in Table 3. The standard deviations of the residuals are $0^{\prime \prime} .10$ and $3^{\circ} .4$ for $\rho$ and $\theta$, respectively. Those values are those expected for a sub-arcsecond couple. A few observations had to be discarded, but convergence was easy to achieve. As already mentioned by Muller (1951) and Baize (1992) there is a systematic difference in separation for the measurements made before and after the periastron passage of 1934. In average, the former are larger and more scattered than the latter.

Using the Hipparcos parallax value revised by Gatewood et al. (2001) of $\pi_{H I P}=13.8 \pm 0.41$ mas, we obtain 
a total system mass of $7.6 \pm 1.6 \mathrm{M}_{\odot}$. This value is in good agreement with the value of $8.8 \pm 0.9 \mathrm{M}_{\odot}$ derived by Gatewood et al. (2001), who performed a detailed study of this system that combined spectroscopic and visual data.

\section{Conclusion}

In the first semester of 2006, we performed 175 observations of 169 visual binaries with PISCO in Merate. When adding those made since 2004, the total reaches 1078 observations, which is more than twice the number of binary observations made with PISCO on the 2-meter Bernard Lyot telescope of Pic du Midi during the period 1993-1998. The new exploitation of PISCO in Merate has thus already provided a significant contribution to the measurements of close visual binary stars.

The measurements reported here have already been used to revise the orbits of ADS 11956 (Scardia et al., 2008b) and of ADS 11479, 11584 and 16538, whose elements are presented in this paper. We hope that our measurements will be useful to the astronomical community and help to improve the accuracy of many other orbits in the future.

Acknowledgements. We thank the members of the United States Naval Observatory, Washington DC, for kindly sending on request some lists of measurements of visual binaries. This work has made use of the "Fourth Catalogue of Interferometric Measurements of Binary Stars" (http://ad.usno. navy.mil/wds/int4), the "Sixth Catalogue of Orbits of Visual Binary Stars" (http://ad.usno.navy.mil/wds/orb6), the Washington Double Star Catalogue maintained at the U.S. Naval Observatory (http://ad.usno.navy.mil/ wds/wds) and the SIMBAD data base operated by the Centre de Données Astronomiques de Strasbourg (France).

\section{References}

Aitken, R.G.: 1932, "New General Catalogue of Double Stars", Carnegie Institute, Washington

Alzner, A.: 1996, IAU Commission 26, Inf. Circ. No 130

Alzner, A.: 2006, IAU Commission 26, Inf. Circ. No 160

Aristidi, E., Carbillet, M., Lyon, J.-F., Aime, C.: 1997, A\&AS, 125, 139

Baize, P.: 1989, IAU Commission 26, Inf. Circ. No 107

Baize, P.: 1992, A\&A Suppl. 92, 31

Balega, I.I., Balega, Yu.Yu., Hofmann, K.-H., Maksimov, A.F., Pluzhnik, E.A., Schertl, D., Shkhagosheva, Z.U., Weigelt, G., 2002, A\&A, 385, 87

Campbell, W.W.: 1901, ApJ 14, 138

Gatewood, G., Han, I., de Jonge, J.K., Reiland, C.T.: 2001, ApJ 549, 1145

Germain, M.E., Douglass, G.G., Worley, C.E.: 1999, AJ 117, 2511

Harper, W.E.: 1925, Publ. Dom. Astrophys. Obs. Victoria, 3,189
Hartkopf, W.I.: \& Mason B.D., 2008, "Sixth Catalogue of Orbits of Visual Binary Stars" http://ad.usno.navy.mil/wds/orb6.html (OC6)

Hartkopf, W.I., Mason, B.D., Wycoff, G.L., McAlister, H.A.: 2008, "Fourth Catalogue of Interferometric Measurements of Binary Stars" http://ad.usno.navy.mil/wds/int4.html (IC4)

Hellerich, J.: 1925, Astron. Nachr. 223, 335

Hopmann, J.: 1973, Astron. Mitt. Wien, 13

Kowalsky, M.: 1873, Procès-verbaux de l'Université Imperiale de Kasan

Mason, B.D., Wycoff, G.L., Hartkopf, W.I.: 2008, "Washington Double Star Catalogue" http://ad.usno.navy.mil/wds/wds.html

Muller, P.: 1951, Bull. Astron. 16, 210

Prieur, J.-L, Koechlin, L., André, C., Gallou, G., Lucuix, C.: 1998, Experimental Astronomy, vol 8, Issue 4, 297

Prieur, J.-L., Carquillat, J.-M., Ginestet, N., Koechlin, L., Lannes, A., Anterrieu, E., Roques, S., Aristidi, E., Scardia, M.: 2003, ApJ Suppl., 144, 263

Prieur, J.L., Scardia, M., Pansecchi, L., Argyle, R.W., Sala, M., Ghigo, M., Koechlin, L., Aristidi, E.: 2008, MNRAS, 387, 772 (Paper V)

Scardia, M., Prieur, J.-L., Aristidi, E., Koechlin, L.: 2000, ApJS, 131, 561

Scardia, M., Prieur, J.-L., Sala, M., Ghigo, M., Koechlin, L., Aristidi, E., Mazzoleni, F.: 2005, MNRAS, 357, 1255 (with erratum in MNRAS 362, 1120) (Paper I)

Scardia, M., Prieur, J.-L., Pansecchi, L., Argyle, R.W., Sala, M., Ghigo, M., Koechlin, L., Aristidi, E.: 2006, MNRAS, 367, 1170 (Paper II)

Scardia, M., Prieur, J.-L., Pansecchi, L., Argyle, R.W., Basso, S., Sala, M., Ghigo, M., Koechlin, L., Aristidi, E.: 2007, MNRAS, 374, 965 (Paper III)

Scardia, M., Prieur, J.-L., Pansecchi, L., Argyle, R.W., Sala, M., Basso, S., Ghigo, M., Koechlin, L., Aristidi, E.: 2008a, Astron. Nach., 329, 1, 54 (Paper IV)

Scardia, M., Prieur, J.-L., Pansecchi, L., Argyle, R.W.: 2008b, Astron. Nach., 329, 4, 379

Scarfe, C.D., Regan, J., Barlow, D., Fekel, F.C.: 1983, MNRAS, 203, 103

Symms, L.S.T.: 1964, IAU Commission 26, Inf. Circ. No 33

Weigelt, G., 1977, Opt. Comm., 21, 55

Worden, S.P., Murray, K.S., Schmidt, G.D., Angel, J.R.P., 1977, Icarus 32, 450

Zulevic, D.J.: 1975, IAU Commission 26, Inf. Circ. No 66 